\title{
Computational Prediction of Pressure and Thermal Environments in the Flame Trench with Launch Vehicles
}

\author{
Christoph Brehm*, Emre Sozer* \\ ${ }^{*}$ Science and Technology Corporation, Moffett Field, CA 94035 \\ Michael F. Barad $\dagger$ Jeffrey A. Housman $\dagger$ Cetin C. Kiris ${ }^{\ddagger}$ \\ ${ }_{\ddagger}$ NASA Ames Research Center, Moffett Field, CA 94035 \\ Shayan Moini-Yekta ${ }^{\S}$

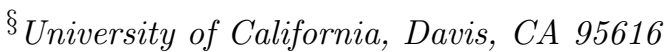 \\ Bruce T. Vu, Christopher R. Parlier" \\ III NASA Kennedy Space Center, FL 32899
}

\begin{abstract}
One of the key objectives for the development of the 21st Century Space Launch Complex is to provide the flexibility needed to support evolving launch vehicles and spacecrafts with enhanced range capacity. The launch complex needs to support various proprietary and commercial vehicles with widely different needs. The design of a multi-purpose main flame deflector supporting many different launch vehicles becomes a very challenging task when considering that even small geometric changes may have a strong impact on the pressure and thermal environment.

The physical and geometric complexity encountered at the launch site require the use of state-of-the-art Computational Fluid Dynamics (CFD) tools to predict the pressure and thermal environments. Due to harsh conditions encountered in the launch environment, currently available CFD methods which are frequently employed for aerodynamic and thermal load predictions in aerospace applications, reach their limits of validity. This paper provides an in-depth discussion on the computational and physical challenges encountered when attempting to provide a detailed description of the flow field in the launch environment. Several modeling aspects, such as viscous versus inviscid calculations, single-species versus multiple-species flow models, and calorically perfect gas versus thermally perfect gas, are discussed. The Space Shuttle and the Falcon Heavy launch vehicles are used to study different engine and geometric configurations. Finally, we provide a discussion on traditional analytical tools which have been used to provide estimates on the expected pressure and thermal loads.
\end{abstract}

\footnotetext{
*Research Scientist, Applied Modeling and Simulation Branch, NAS Division, MS N258-2 and AIAA Senior Member

${ }^{\dagger}$ Research Scientist, Applied Modeling and Simulation Branch, NAS Division, MS N258-2 and AIAA Senior Member

${ }^{\ddagger}$ Branch Chief, Applied Modeling and Simulation Branch, NAS Division, MS N258-2 and AIAA Senior Member

$\S$ PhD Candidate, Mechanical and Aeronautical Engineering, University of California, Davis, and AIAA Student Member.

ILead AST, Gas and Fluid Systems, AIAA Senior Member

$\|$ AST, Experimental Facilities Technq. and AIAA Senior Member
} 


\section{Introduction}

With the retirement of the Space Shuttle, NASA is developing a new heavy-lift capability, the Space Launch System (SLS). In addition, commercial vehicles such as SpaceX's Falcon, ATK's Liberty and ULA's Delta IV Heavy and Atlas V Rockets, are also being considered for transporting crew/cargo to the International Space Station (ISS). To accommodate launches of these vehicles, Kennedy Space Center (KSC) is re-assessing their launch site capabilities. Components of particular interest are the Flame Trench and the Main Flame Deflector (MFD) whose main purpose is to safely deflect the exhaust plume away from the vehicle as it lifts off.

Re-design of the MFD requires assessment of its performance regarding contain- ment of the plume in the trench, pressure and thermal loads on the MFD and the surrounding environment for the launch of SLS and commercial vehicles. A Computational Fluid Dynamics (CFD) approach is well suited to provide the necessary predictive capabilities during a rapid design cycle. Through CFD, detailed examination of the concern areas, incremental design trade studies and comparative studies of various MFD concepts can be achieved in a time and cost efficient manner.

This paper will discuss the challenges encountered when faced with the task of making accurate predictions in the launch environment. Accurate predicitions of this challenging environment are necessary to ensure a safe launch of the vehicle. Moreover, damages to the launch pad after each rocket launch must be minimized in order to reduce maintenance cost. Upon ignition of the Solid Rocket Boosters (SRBs), strong Ignition Over-Pressure (IOP) waves are generated while the thrust is building up. The sudden injection of mass into confined regions cause the formation of compression and expansion waves, which reflect off the different surfaces, e.g. mobile launcher and MFD. One of the potential risks of reflected IOP waves is that these waves travel back towards the vehicle and may damage the payload or the structure of the vehicle itself. The main purpose of the water sound suppression system is to protect the orbiter and its payloads from being damaged by reflected high-energy waves. In order to make the water sound suppression system highly effective the properties of the IOP waves, such as directionality and magnitude, need to be well understood.

A particular challenge for the CFD modeling of the launch environment is the large range of physically relevant length scales involved, and the time dependent nature of the regions of interest. Some regions of the flow may be appropriately modelled as inviscid flow regions and in other regions viscous terms and boundary layer resolution are required for the study of the thermal environment. Ensuring sufficient resolution of the physics through a uniformly fine grid is a prohibitively expensive approach due to the scale of the problem. Locally refined manual gridding approaches require a-priori knowledge of the critical parts of the flow and is unsuitable to effectively utilize in problems with large temporal variations. The potential geometric complexity that may be required for launch environment simulations also renders a structured body-fitted grid generation approach to be unpractical, at least during a rapid design cycle.

The current abstract provides a rough overview on what can be expected in the final paper. The first part of this abstract will describe the computational methods which are used for simulating the launch environment. Next, two validation cases cosindering the Space Shuttle launch vehicle are utilized to demonstrate the capabilities of our in-house simulation tools. The last part of this abstract will focus on providing an outlook on the final paper. Some of the current challenges for the CFD analysis are discussed and preliminary results for the Falcon Heavy launch vehicle are presented. In contrast to related work presented in Moini-Yekta et $a l .,{ }^{1}$ the final paper will provide a more detailed discussion about the ongoing physics in launch environment instead of validating the current hybrid LAVA-structured/LAVA-unstructured approach. Moreover, some more advanced strategies for analyzing the unsteady flow data based on discrete Fourier transforms and Proper Orthogonal Decomposition (POD) will be used.

\section{Computational Methods}

Our computational framework, called Launch Ascent and Vehicle Aerodynamics (LAVA), contains two main components to overcome the difficulties related to the geometric complexity of the launch site. The first component is LAVA-Cartesian, a block-structured, immersed-boundary Cartesian code with Adaptive Mesh Refinement (AMR) capability. The second component is LAVA-Unstructured, an unstructured finite volume solver, which is utilized to model the near wall viscous and thermal effects. The unstructured grid can be generated with significantly less time than previously achieved when employing structured body-fitted grids. 


\section{II.A. LAVA-Cartesian}

LAVA-Cartesian is a block-structured, Immersed Boundary (IB) AMR code. This methodology is capable of automatically generating, refining, and coarsening nested Cartesian volumes. LAVA's AMR-IB method is designed to automatically generate the volume grids from a closed surface triangulation, and dynamically track important flow features as they develop.

AMR is a proven methodology for multi-scale problems with an extensive existing mathematical and software knowledge base. ${ }^{2-6}$ The code has been extended using data structures and inter-level operators from the high-performance Chombo AMR library ${ }^{7}$ to provide a multi-resolution capability that can coarsen and refine the grid locally as a simulation progresses (Figure 1a). A sharp immersed-boundary representation with ghost cell boundary treatment ${ }^{8}$ and automatic grid generation requiring only a surface triangulation make it possible to easily model complex geometry.

In this code the compressible Reynolds-Averaged Navier-Stokes (RANS) equations are solved with multispecies (no chemical reactions) and unsteady capabilities using dual-time stepping.

LAVA-Cartesian simulations presented herein are conducted using inviscid equations due to the high cost of resolving boundary layers with isotropic Cartesian cells. In this paper we will investigate if the inviscid approach is sufficient in order to accurately predict pressure environment. Viscous dominated flow features such as the thermal loading on the MFD and trench walls are investigated using the LAVA-Unstructured code where grid points are clustered in regions of interest regions of interest, i.e. highly-stretched meshes are utilized to capture the boundary layer

\section{II.B. LAVA-Unstructured}

LAVA-Unstructured is a body-fitted code that can utilize arbitrary polyhedral cell type grids. The code uses a cell-centered finite volume formulation with a dual-time stepping scheme. Currently, the solver is second-order accurate in space and time.

The compressible RANS equations are solved with the Spalart-Allmaras (SA) ${ }^{9}$ or Shear Stress Transport $(\mathrm{SST})^{10}$ turbulence models.

Unstructured, arbitrary cell type grids for complex geometries can be generated rapidly with minimal user effort. With the utilization of near-body prism layers, boundary layers can be resolved accurately and efficiently. The unstructured grid can also be coupled with a Cartesian grid using an overset methodology. A hybrid approach takes advantage of the benefits of both grid types and the LAVA framework. Figure 1b illustrates the overlap between the near-body unstructured grid and the off-body Cartesian grid as well as the buffer region included for accuracy.

LAVA-Unstructured code has the capability to perform conjugate simulation of fluid flow and heat transfer through solid boundaries for realistic, unsteady surface temperature and heat rate predictions. The solid is modeled through local 1-dimensional rays along the surface normal direction at each grid face on the surface. Temperature dependent solid material thermal properties can be used where data is available. A graphical representation of the conjugate heat transfer method is shown in Figure 1c.

\section{II.B.1. Surface Material Modeling}

In the LAVA-Unstructured thermal simulations presented herein, the MFD surface and in some cases the trench walls are assumed to be coated with the Fondu Fyre WA-1G material with properties summarized in Table 1. ${ }^{11}$ Fondu Fyre WA-1G is manufactured by Pryor Giggey Co., 2501 Alexandria Rd, Anniston, AL 36201 .

\begin{tabular}{|c|c|c|}
\hline Property & Value & Units \\
\hline Density & 2000 & $\mathrm{~kg} / \mathrm{m}^{3}$ \\
\hline Thermal Conductivity & 1 & $\mathrm{~W} / \mathrm{m} . \mathrm{K}$ \\
\hline Specific Heat & $600-1600$ (Temperature dependent) & $\mathrm{J} / \mathrm{kg} . \mathrm{K}$ \\
\hline Thickness & 6 & inches \\
\hline Melting Temperature & 1373 & $\mathrm{~K}$ \\
\hline
\end{tabular}

Table 1. Properties used for Fondu Fyre WA-1G material ${ }^{11}$ 


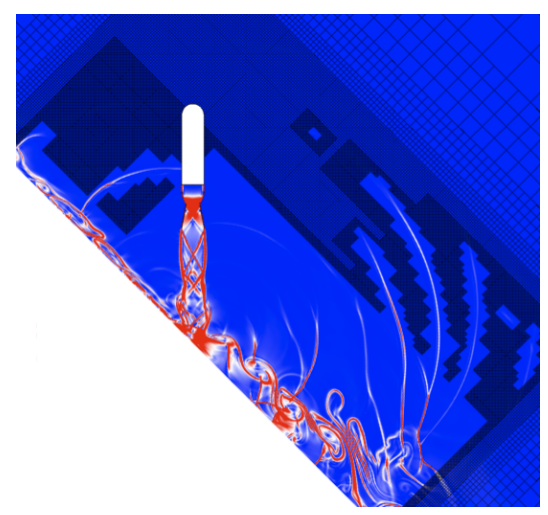

(a)

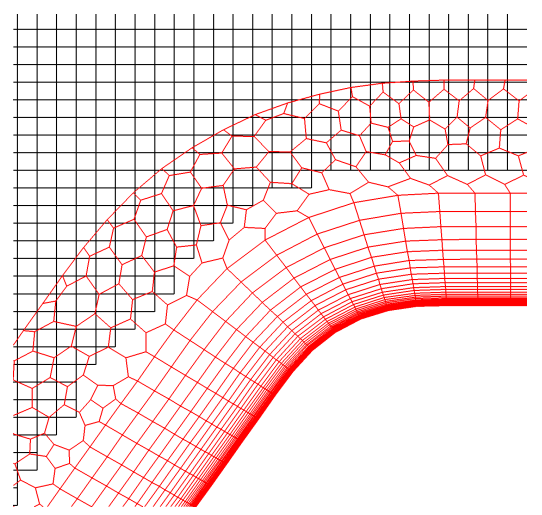

(b)

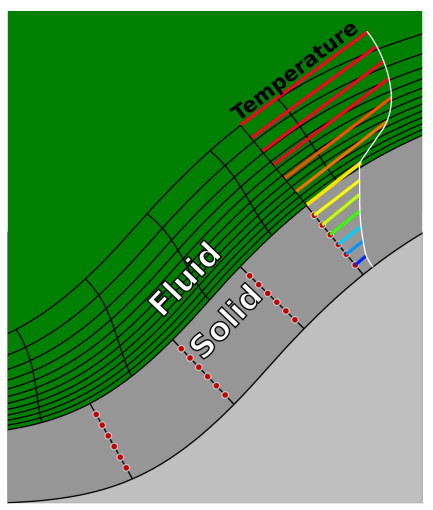

(c)

Figure 1. Highlights of LAVA framework features: (a) block-structured Cartesian AMR feature tracking, (b) hybrid grid coupling of Cartesian and unstructured grids via overset and (c) schematic of conjugate heat transfer method between body-fitted-unstructured and $1 \mathrm{D}$ solid grids.

Note that the surface material temperature is capped at the melting point. Phase change and erosion are not modeled. Unless otherwise stated, the temperature at the back of the material is held constant at $300 \mathrm{~K}$.

\section{Verification and Validation Results}

Two verification and validation cases were chosen to demonstrate the capabilities of the current LAVA framework. For more information about the analysis about the test cases see. ${ }^{1}$

\section{III.A. 2D Trench Test Case}

A preliminary 2D trench test case is used to establish the spatial and physical modeling requirements for the launch environment simulations. The performance of different modeling approaches is analyzed by performing inviscid single gas and multiple-species as well as viscous unstructured and hybrid approaches. To assess the different approaches, code-to-code comparisons are done using OVERFLOW ${ }^{12}$ and the LAVA framework. OVERFLOW solves the time-dependent, Reynolds-averaged, compressible Navier-Stokes equations employing multiple overset structured grids.

\section{III.A.1. Problem Setup}

To emulate the launch environment, the 2D trench test problem, which consists of a supersonic jet impinging on a MFD, is created. A simplified Mobile Launch Platform (MLP) is also included in the geometry to incorporate the confinement effect of the exhaust holes. Unsteady pressure conditions are specified at the nozzle exit with a hyperbolic tangent ramping function to mimic engine conditions. A rectangular domain is used with outer boundaries set to 200 times the nozzle diameter. For the inviscid simulations all surfaces are set to slip boundary conditions, while in the viscous simulations the MFD and a small region around it are set to no-slip boundary conditions (the red region in Figure 2c). Simulations are performed for a total duration of 2.0 seconds with a time step of $3.5 \times 10^{-5}$ based on best practices for $3 \mathrm{D}$ simulations. ${ }^{13,14}$

With the 2D computational setup, the unsteadiness and shock structures are expected to be different but display qualitatively similar behavior as one may expect in the 3D simulations. To monitor the flow, 28 point probes are placed near the plume impingement location. All simulations were run unsteady with dual-time stepping and second-order spatial and temporal discretization. The LAVA codes were run with 30 sub-iterations based on best practices and the OVERFLOW simulation was run with 100 sub-iterations based on a sub-iteration study. Both LAVA-Cartesian/Unstructured and OVERFLOW used the SpalartAllmaras RANS turbulence model. An in-depth study of space-time convergence was previously discussed by Housman ${ }^{15}$ on a similar $2 \mathrm{D}$ test case. 


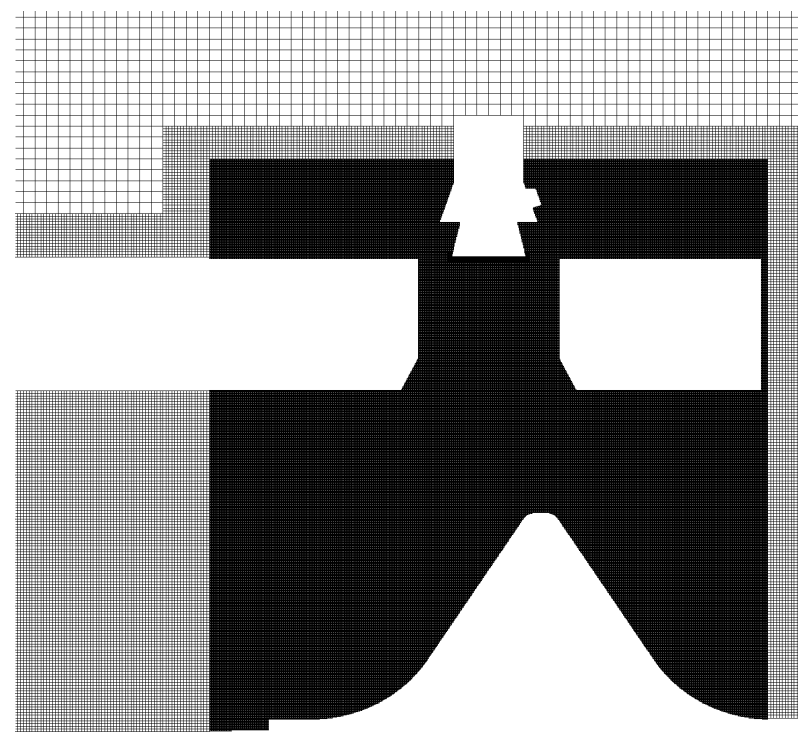

(a) LAVA-Cartesian

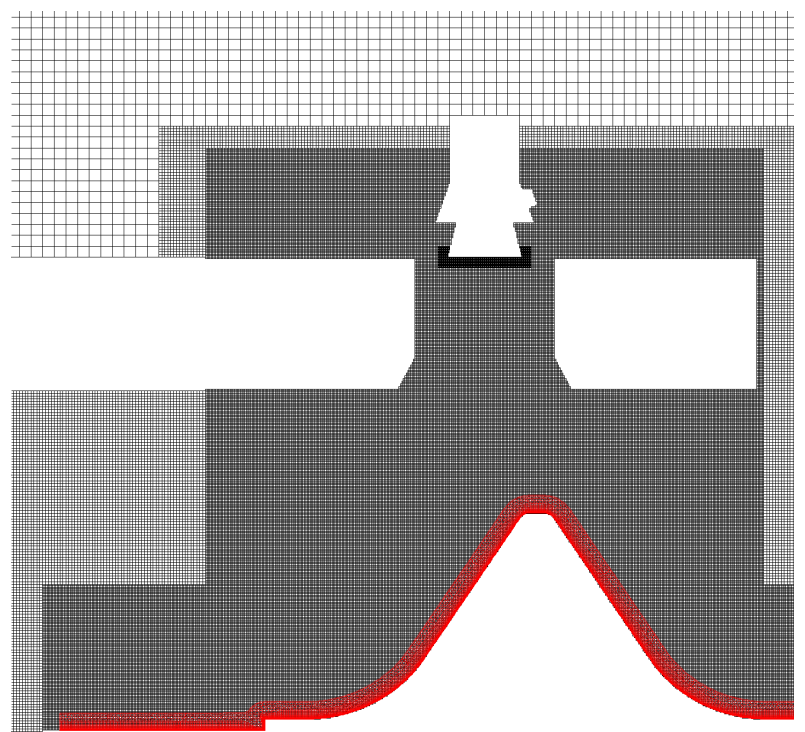

(c) LAVA-Cartesian/Unstructured

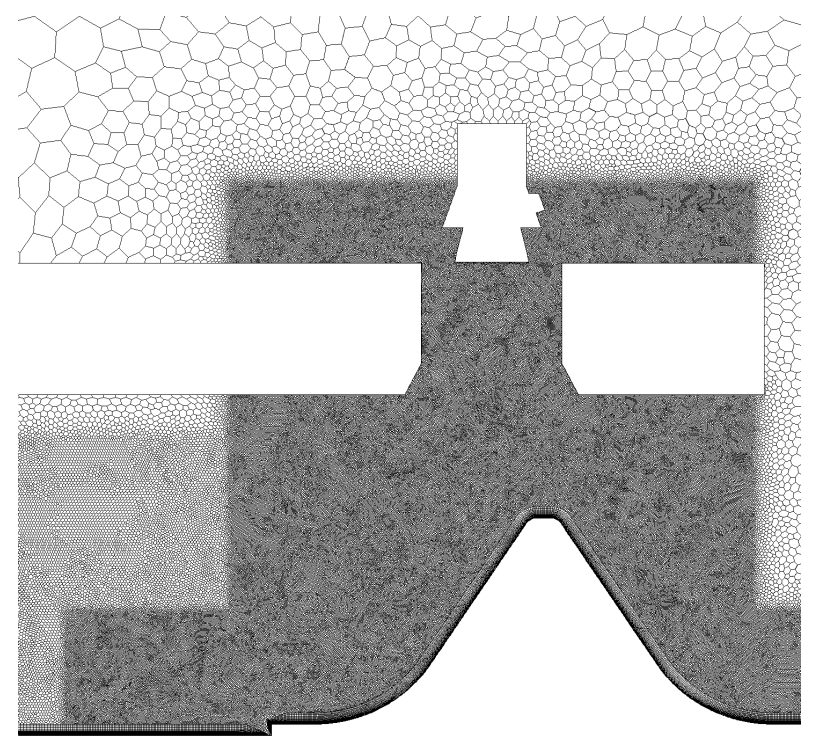

(b) LAVA-Unstructured

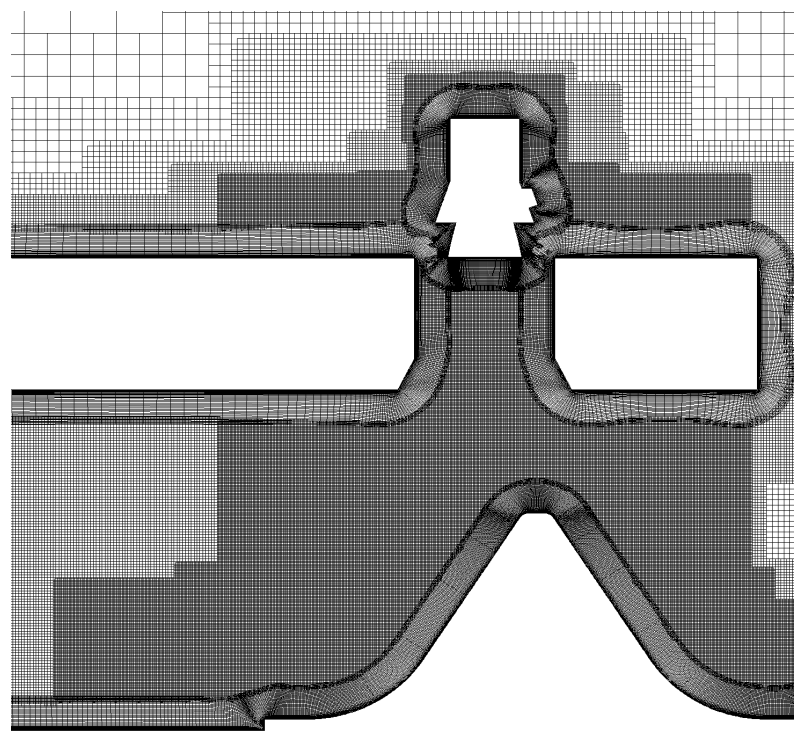

(d) OVERFLOW

Figure 2. Computational grid for 2D trench test case for (a) LAVA-Cartesian, (b) LAVA-Unstructured, (c) LAVACartesian/Unstructured (unstructured shown in red) and (d) OVERFLOW.

\section{III.A.2. Computational Grids}

The generation of the 2D volume grid requires several gridding strategies to be implemented. A plume grid spacing of $15 \mathrm{~cm}$ was targeted for the plume and main flame deflector region to resolve the flow structure. Additional resolution was placed on the North side of the flame trench to track flow features. The Cartesian mesh is shown in Figure 2a and uses a seven level AMR grid with approximately 850k cells. Refinement boxes were manually specified around the nozzle, plume region and MFD. The unstructured grid emulates the Cartesian mesh but is coarsened away from the MFD to reduce the cell count. Figure $2 \mathrm{~b}$ shows the prismatic/polyhedral mesh which features viscous spacing $\left(2.0 \times 10^{-6}\right.$ wall spacing for a $\left.y^{+} \approx 1\right)$ on the MFD and $292 \mathrm{k}$ cells.

The hybrid grid seeks to combine the unstructured viscous wall spacing on the MFD with the efficient AMR off-body of block-structured grids. Hence, a viscous unstructured prismatic/polyhedral grid is specified within a 1 meter region of the MFD surface with a block-structured Cartesian off-body mesh and immersed- 


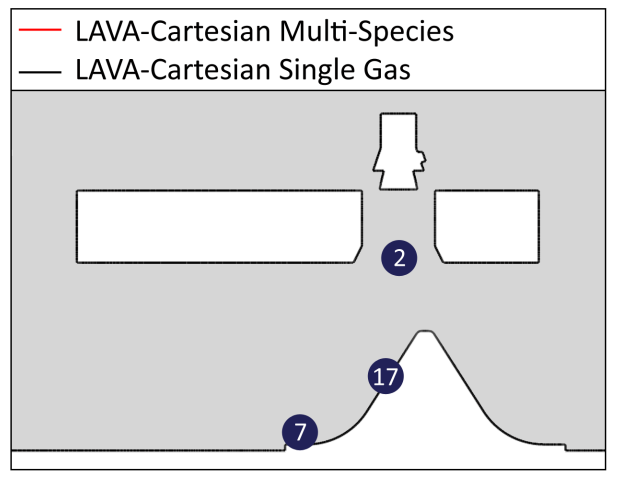

(a) Schematic

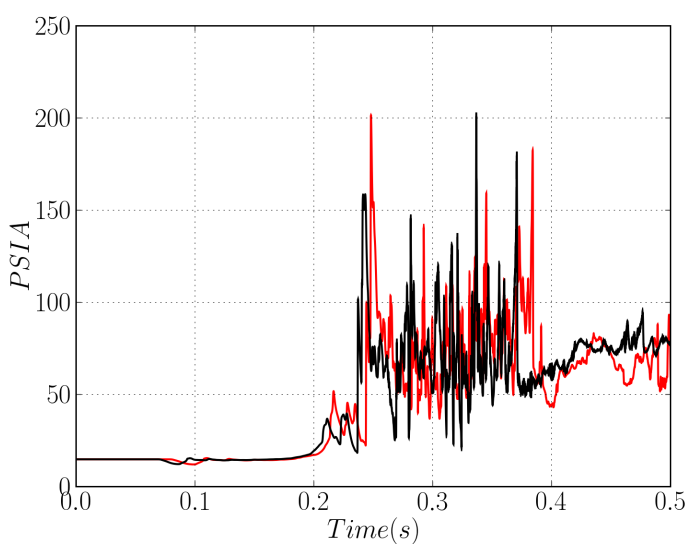

(c) Point 7

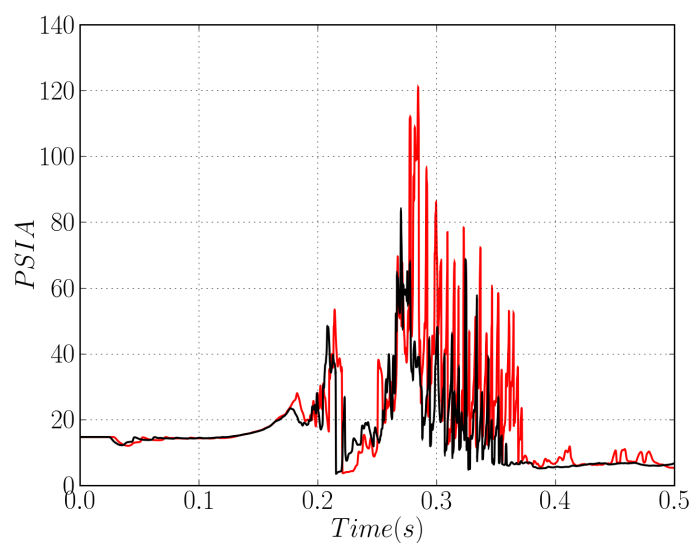

(b) Point 2

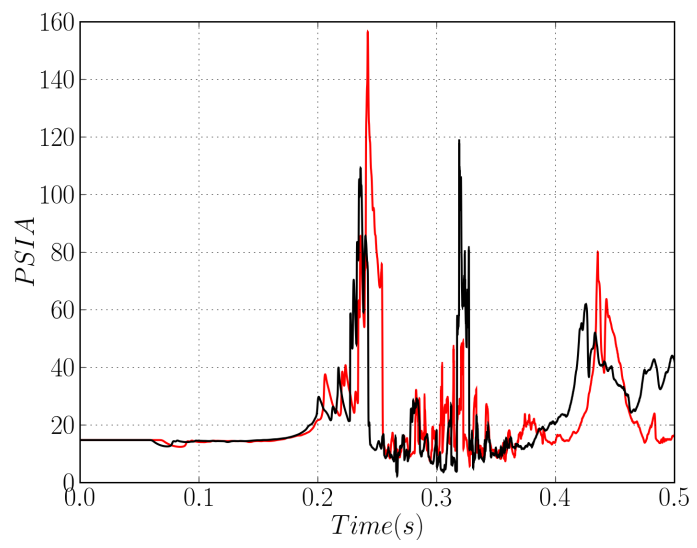

(d) Point 17

Figure 3. Unsteady point probe pressure history: (a) schematic of the point sample locations, at (b) point 2, (c) point 7 and (d) point 17 for LAVA-Cartesian inviscid single gas and multi-species.

boundary treatment on the other surfaces. The grid is shown in Figure 2c and contains 397k Cartesian cells and 30k unstructured cells. The OVERFLOW grid is also shown in Figure $2 \mathrm{~d}$ and contains 640k grid points with 321 grid zones. Viscous spacing is also specified on the MFD with similar resolution in the plume and trench.

\section{III.A.3. Results}

In this section we provide $2 \mathrm{D}$ simulation results and a preliminary discussion on the different aspects which will be discussed for the 3D case in the final paper. The first aspect is the sensitivity of single gas versus multi-species simulations for the pressure environment. Simulations are employed using LAVA-Cartesian with inviscid single gas and multi-species models. For the multi-species simulations we assume a homogeneous mixture model for the gas applying Amagat's law. The unsteady pressure histories for three numerical sensors (2, 7 and 17) are shown in Figure 3. These points were selected to analyze the pressure history inside the jet plume, near the first impingement location and near the termination of the Mach diamond at the bottom of the deflector. The pressure signals at all three numerical sensors for the single gas and multi-species simulations are very similar. However, a significant difference in the magnitude of the IOP wave between the different gas models can be observed at points 7 and 17. In addition, a slight phase shift is evident due to apparent differences in wave propagation speed. This can simply be explained by considering that the gas mixture is modeled as an ideal gas in the current simulations and a reduction in the heat capacity ratio for the multi-species simulations lowers the speed of sound. In the final conference paper a more detailed analysis of the unsteady time signal will be provided. 
A second modeling aspect that is concerned with the effect of viscoscity on the pressure environment. A comparison of the instantaneous Mach number contours at $t=0.4 \mathrm{~s}$ after SRB ignition for the LAVACartesian multi-species, LAVA-Unstructured, LAVA-Cartesian/Unstructured and OVERFLOW simulations are presented in Figure 4. Even though the basic flow features should be quiet similar between the four simulations with the plume being primarily deflected to the North side (shown to the left on the images) of the trench and shock structures near the step of the deflector, some differences in the simulations are apparent. The higher resolution of the LAVA-Cartesian and OVERFLOW simulations is evident by the finer flow structures in the trench. The preliminary hybrid LAVA-Cartesian/Unstructured simulation compares well with the individual solvers and OVERFLOW. The viscous effects which are likely responsible for the differences between the LAVA-Cartesian and LAVA-Unstructured simulations will be further analyzed in the final paper.

To assess the accuracy of the hybrid approach, the unsteady pressure probes were compared between LAVA-Cartesian/Unstructured and OVERFLOW. Three points were selected to capture some details of the flow physics: inside the plume (point 2), near the first impingement (point 18) and near the recirculation region at the bottom of the MFD (point 9). Unsteady pressure signals for the two solvers are compared in Figure 5. Higher frequency oscillations are present in the OVERFLOW solution which may be due to the higher grid resolution in comparison to LAVA-Cartesian/Unstructured. However, the general trends in the time-signals for points 2 and 9 are similar, such as the magnitude of the IOP wave at the sensor locations and the general wave pattern picked up by the numerical sensors. At point 18, the initial IOP wave magnitude compares well between the two codes but the unsteady larger scale modulation of the time signal appears to be different. Additional grid refinement and time step subiteration studies will be performed to determine the reason for this discrepancy.

Since heat transfer plays a crucial role in main flame deflector analysis, temperature predictions are important. Figure 6 displays the unsteady temperature history for point probes 2, 9 and 18 for LAVACartesian/Unstructured and OVERFLOW. In the plume, a higher initial temperature peak at point 2 is evident in OVERFLOW, which may be associated with the higher grid resolution. Following the initial peak, a similar trend in the temperature signal can be seen for the two solvers. At this point, it must be emphasized that small differences in the flow field may lead to widely different time-signals when for example a numerical point sensor is located too close to an unsteady shock. As one may expect, similar trends between the two solvers as for pressure signals are also observed at point 9 and 18. Overall the comparison between the hybrid LAVA-Cartesian/Unstructured and OVERFLOW simulations is satisfactory. As mentioned before, additional simulations will be conducted with better matching grid resolutions to investigate the higher frequency discrepancies. 


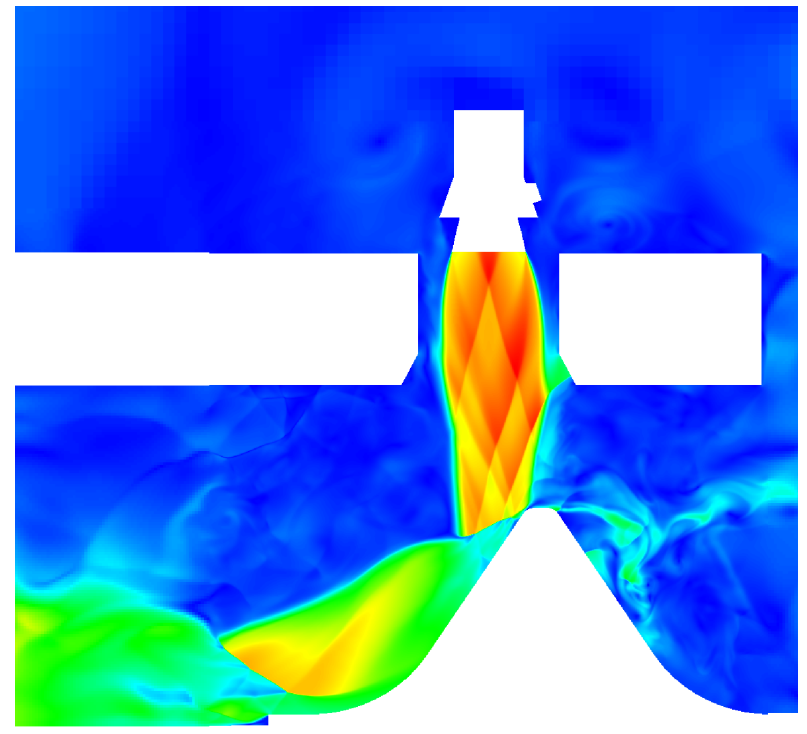

(a) LAVA-Cartesian

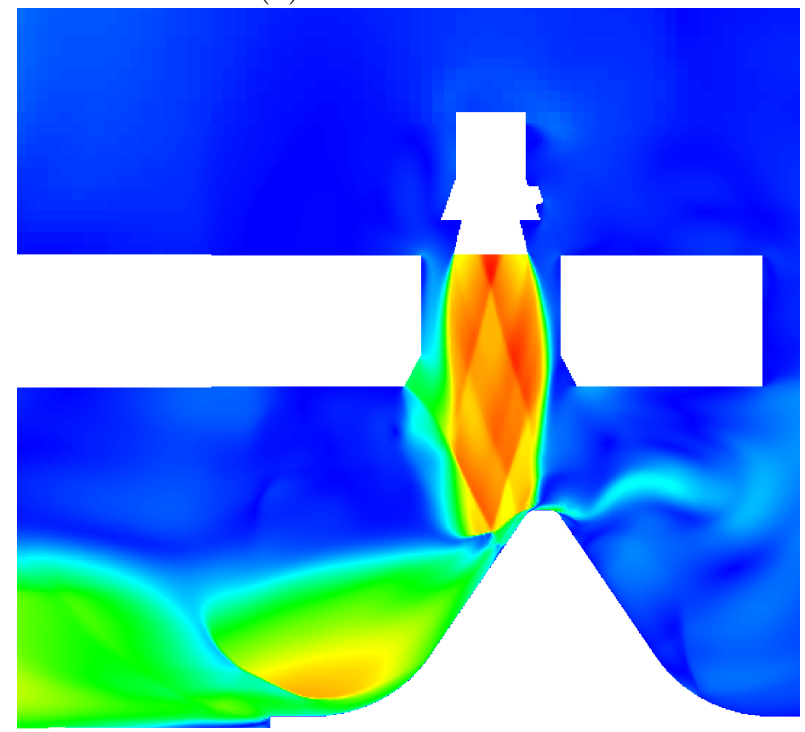

(c) LAVA-Cartesian/Unstructured

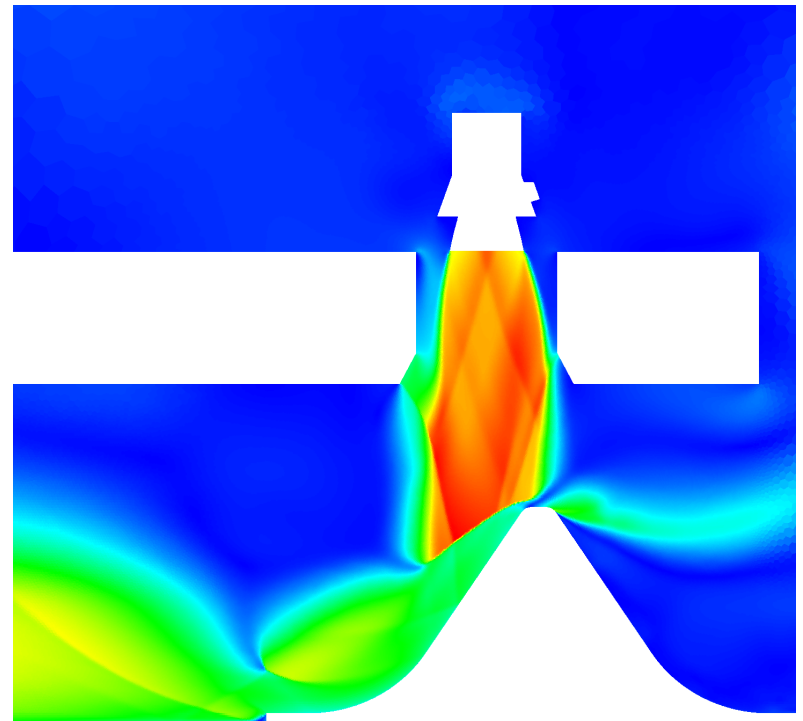

(b) LAVA-Unstructured

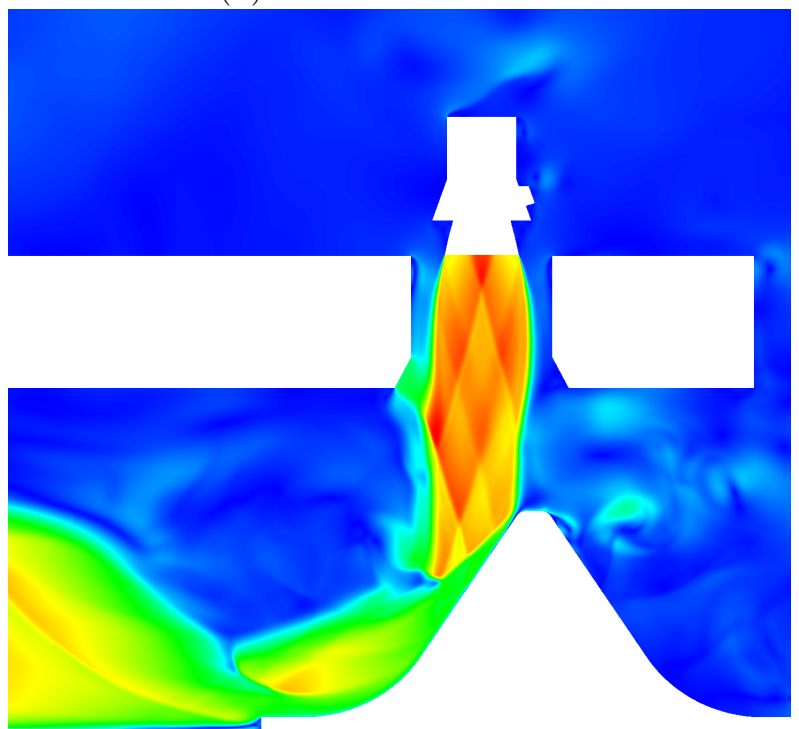

(d) OVERFLOW

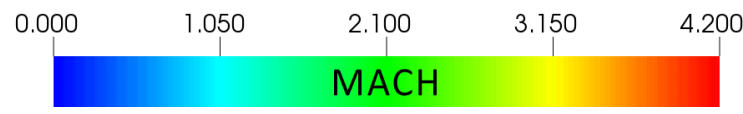

Figure 4. Instantaneous Mach distributions at $t=0.4 \mathrm{~s}$ for $2 \mathrm{D}$ trench test case using (a) LAVA-Cartesian, (b) LAVAUnstructured, (c) LAVA-Cartesian/Unstructured and (d) OVERFLOW. 


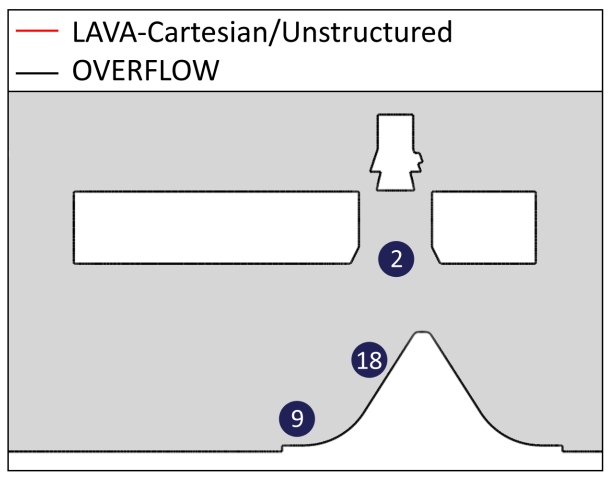

(a) Schematic

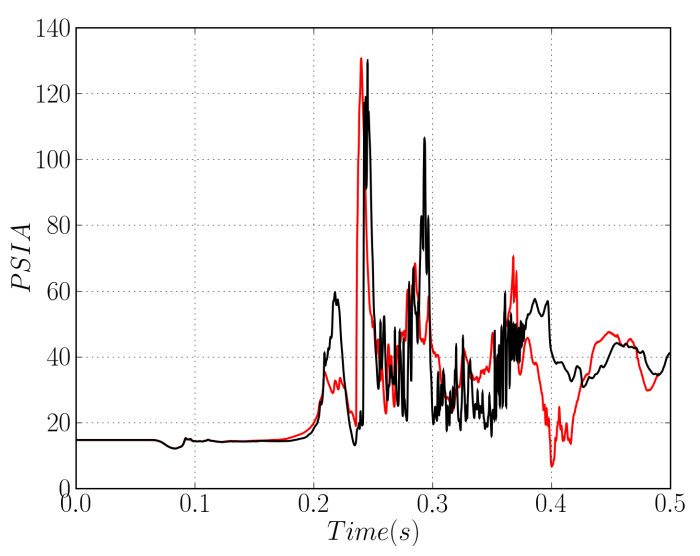

(c) Point 9

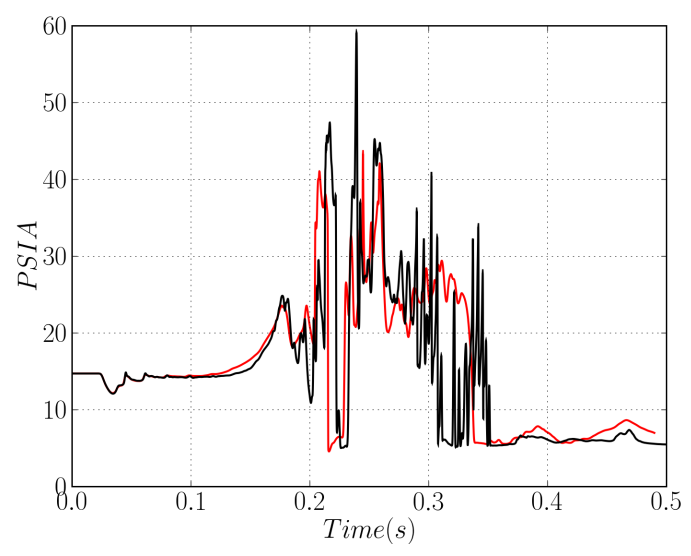

(b) Point 2

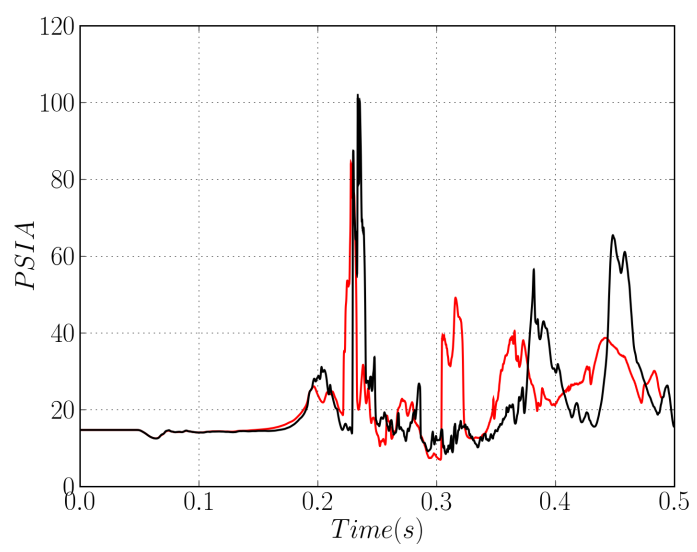

(d) Point 18

Figure 5. Unsteady point probe pressure history: (a) schematic of the point sample locations, at (b) point 2, (c) point 9 and (d) point 18 for LAVA-Cartesian/Unstructured and OVERFLOW. 


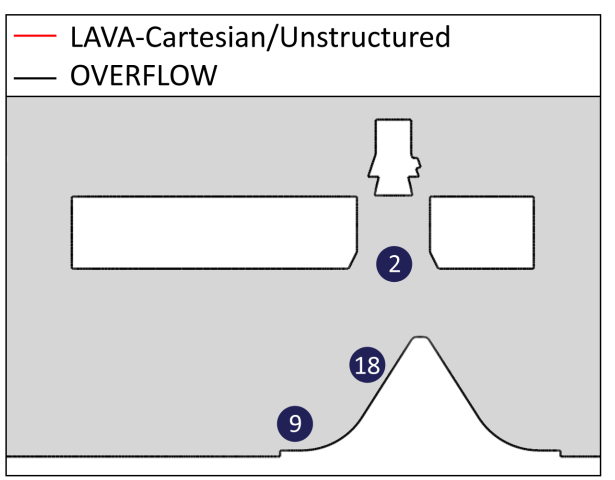

(a) Schematic

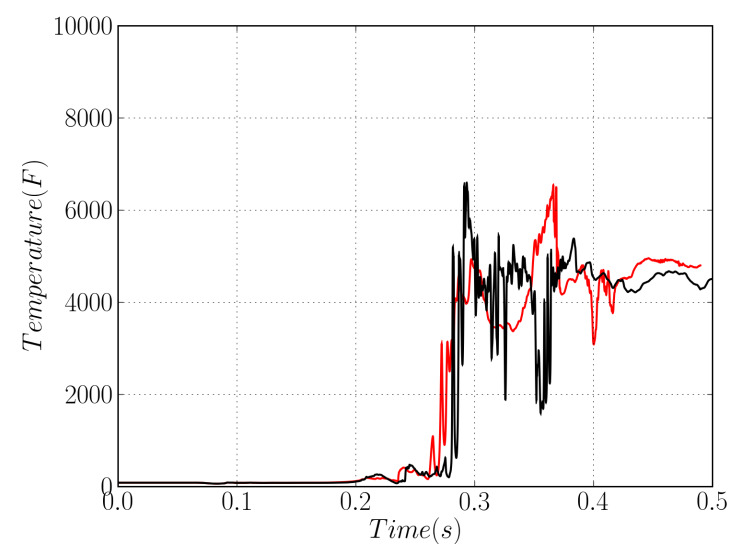

(c) Point 9

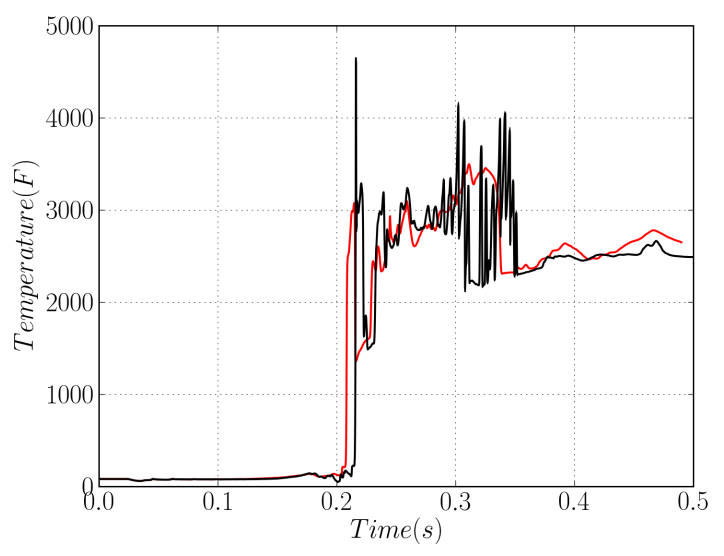

(b) Point 2

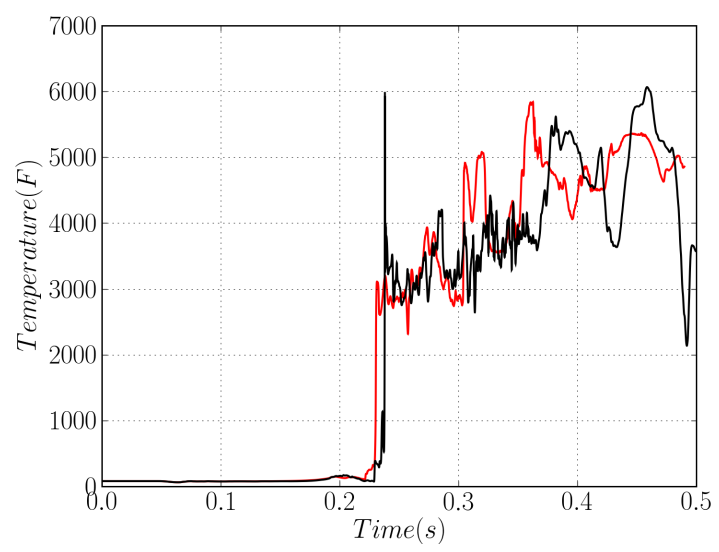

(d) Point 18

Figure 6. Unsteady point probe temperature history: (a) schematic of the point sample locations, at (b) point 2, (c) point 9 and (d) point 18 for LAVA-Cartesian/Unstructured and OVERFLOW. 

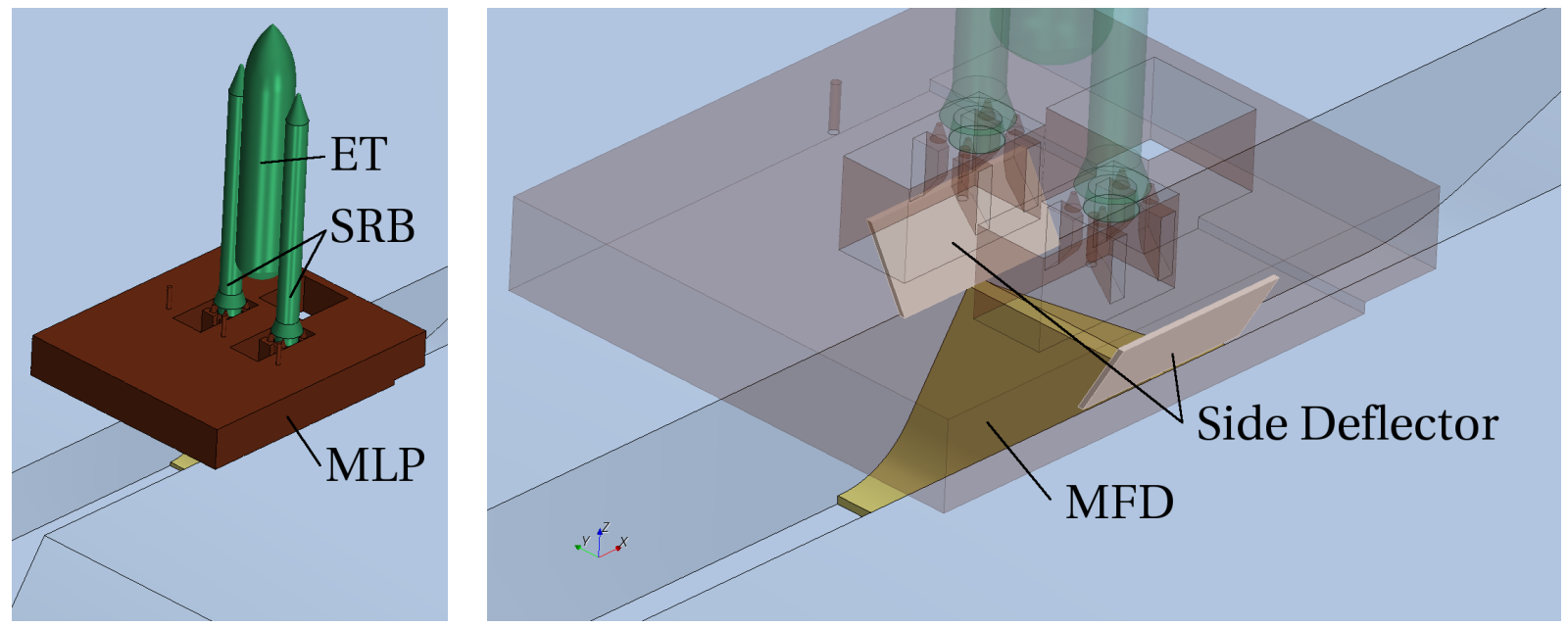

Figure 7. Overview of the computational setup for the STS-135 validation case.

\section{III.B. Space Shuttle Validation Case (STS-135)}

\section{III.B.1. Problem Setup}

The computational geometry for the STS-135 validation test includes the flame trench, surrounding ground terrain, MLP, side deflectors and the launch vehicle. Figure 7 shows an overall schematic of the STS-135 geometry. The flame trench is designed to divert the jet plume and potential debris away from the vehicle. The main component of the flame trench is the MFD located underneath the vehicle nozzles. A simplified vehicle consisting of the external tank (ET) and two SRBs are used in the simulations. The main focus of this work is the IOP environment induced by the SRBs near the MFD, hence a simplified setup is used which excludes the tower and orbiter.

A physical time step of $3.5 \times 10^{-5}$ seconds with 30 sub-iterations are chosen based on previous experience. The test case is modeled up to 1.4 seconds to capture the IOP wave and plume development. During the simulation time, the vehicle is assumed to be static (the actual vehicle moves only a few feet in that time) and the water sound suppression system is not modeled due to the lack of multi-phase capability of the current software. Unsteady boundary conditions are applied at the nozzle exits using the data from the STS-1 launch. The STS-1 boundary conditions have similar conditions as the STS-135, which were not available at the time of this work. The individual solvers LAVA-Cartesian and LAVA-Unstructured were applied to this problem for validation.

LAVA-Cartesian was run with a static mesh whereby the initial mesh was refined in regions where highgradients are expected, such as the exhaust plume, nozzle exits, and the MFD. Viscous LAVA-Unstructured simulations were completed with the SST turbulence model and a conjugate heat transfer method was used on the MFD. A six inch thick Fondu Fyre coating was assumed on the MFD to emulate the STS-135

launch conditions. Surface temperatures were capped at the melting temperature of the material which is approximately 2000 degrees Fahrenheit.

\section{III.B.2. Flight Data}

As a part of an investigation of the refractory material coating on the MFD, the north side of the MFD was instrumented to record pressure, temperature, acceleration and heat rates. Flight data from the commercialoff-the-shelf (COTS) sensors for the STS-135 launch was used for validation. ${ }^{16,17}$ The sensor locations are illustrated in Figure 8 on the North side of the MFD. The COTS sensors feature Kulite pressure transducers, medtherms and erodible Nanmac thermocouples. The sensor data was filtered using a low pass filter at 50 $\mathrm{Hz}$. Only the pressure sensor data is compared to the simulation results in this abstract. For a more detailed discussion on the comparison between the simulation results and the flight data see. ${ }^{1}$ 


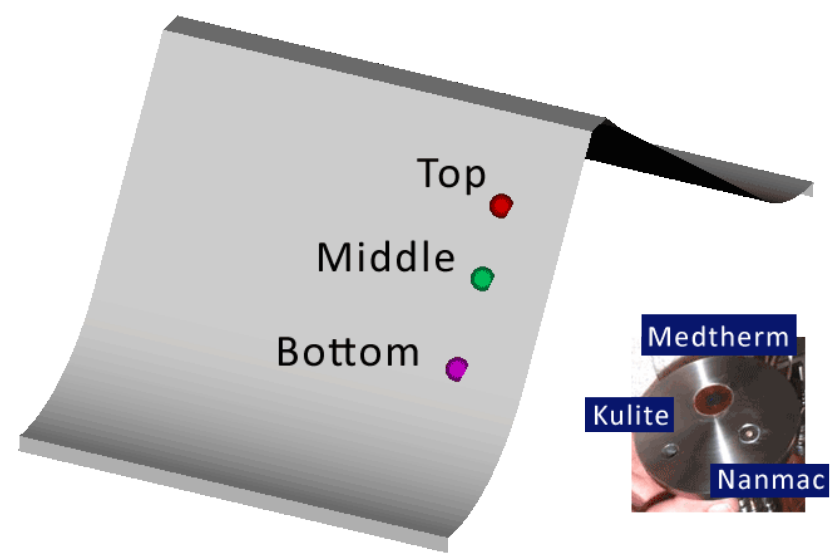

Figure 8. Experimental instrumentation points for North side of main deflector and an image of COTS sensor configuration for STS-135 launch.

\section{III.B.3. Results: Pressure Environment}

With the unsteady ignition of the SRBs, a large ignition over-pressure is generated followed by the impingement of the plume on the MFD. Throughout the development of the flow, multiple shocks are generated on the MFD as the supersonic flow is deflected. This leads to multiple regions of high pressure (impingement points) and low pressure as the plume develops.

The interaction of the two plumes with the MFD and trench leads to a complex and unsteady pressure environment. A key aspect of the final paper is to investigate the required fidelity to accurately predict the pressure environment of this flow field. The LAVA-Cartesian results are obtained by solving the inviscid Euler equations due to the computational advantages of the approach. Inviscid physics dominate the flow, due to the fact that within the nozzle and along the body the boundary layers are generally thin. ${ }^{18}$ In order to investigate the viscous and turbulent effects on the MFD the LAVA-Unstructured with the SST turbulence model was also applied.

The pressure and Mach number distributions shown in Figure 9 illustrate the highly unsteady and shocked flow for LAVA-Cartesian multiple species and single gas as well as single gas LAVA-Unstructured simulations. For all single-gas simulations presented in this section, the exhaust gas properties were used throughout the domain. The results are taken at the quasi-steady state full thrust conditions ( $t=0.7$ seconds) with the contour levels of the Mach number set from 0 to 4.2 and the pressure contour range from -10 to 70 PSIG. Sonic lines are also shown in the PSIG distribution plots to visualize the shock structure on the MFD. The first observation of the results is that the major structures are consistent between each modeling fidelity level. Mach cones within the jet are sharply defined and the strong shock at the first impingement point are captured. Aside from the higher jet spreading rate, which may partially be due to turbulent mixing, the viscous and inviscid results are consistent. Similarly, the single gas and multi-species results have nearly identical pressure values and shock structures. The final paper will present a more detailed comparison between the different modeling aspects, i.e., single-species versus multi-species and viscous versus inviscid.

For a quantitative comparison, unsteady pressure probe data was accumulated for the simulation data and flight data. As shown in Figure 8, three locations (bottom, middle and top) were examined on the MFD. Figure 10 shows the unsteady pressure history for the inviscid multi-species, viscous single-species and both the raw and filtered flight data. Comparison of the simulation data to the flight data at the top sensor reveals an approximately 0.1 second phase lag. The source of the phase lag may be attributed to neglecting the multi-phase effects of the water sound suppression system which would slow the plume propagation speed. Another potential cause of the time lag is differences in the ramping of the STS-1 and STS-135 engine conditions. Small discrepancies in the unsteady pressure ramping has the potential to change the plume characteristics. The numerical simulations also do not account for the multi-phase flow and the fact that waves propagate at different speeds in different mediums. The pressure peaks and the general trends of the unsteady pressure signal, e.g., initial IOP and the following reduction in pressure, are well captured in the CFD simulations. Note, the unfiltered pressure sensor flight data contains high frequency content that is eliminated with filtering. 

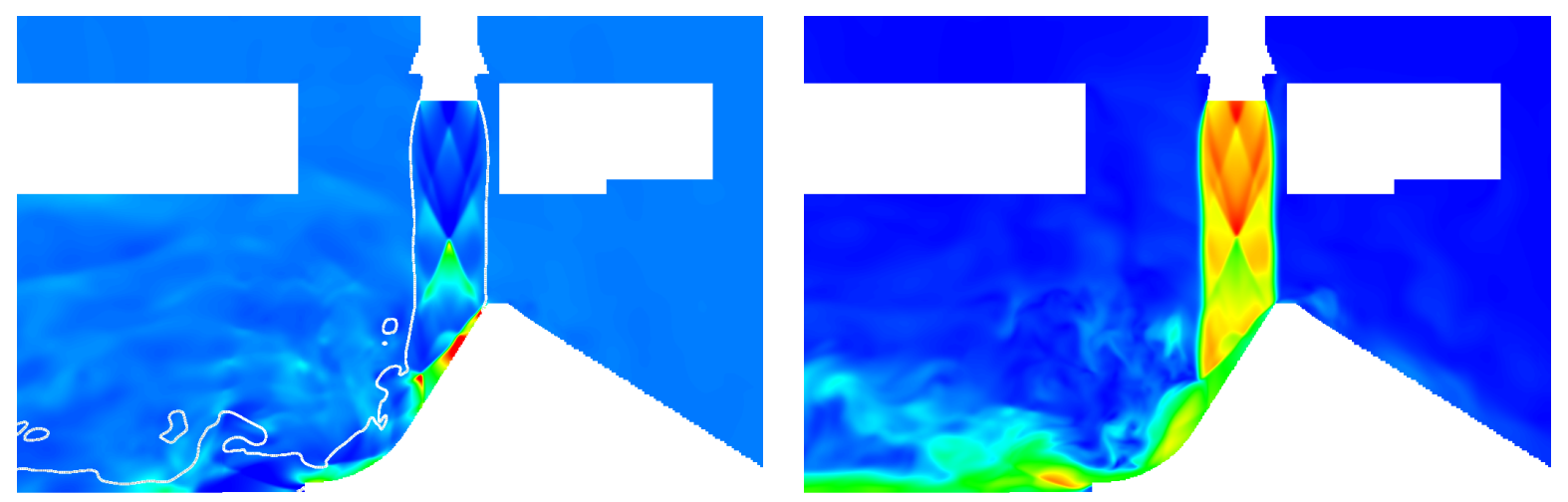

(a) LAVA-Cartesian Multi-Species
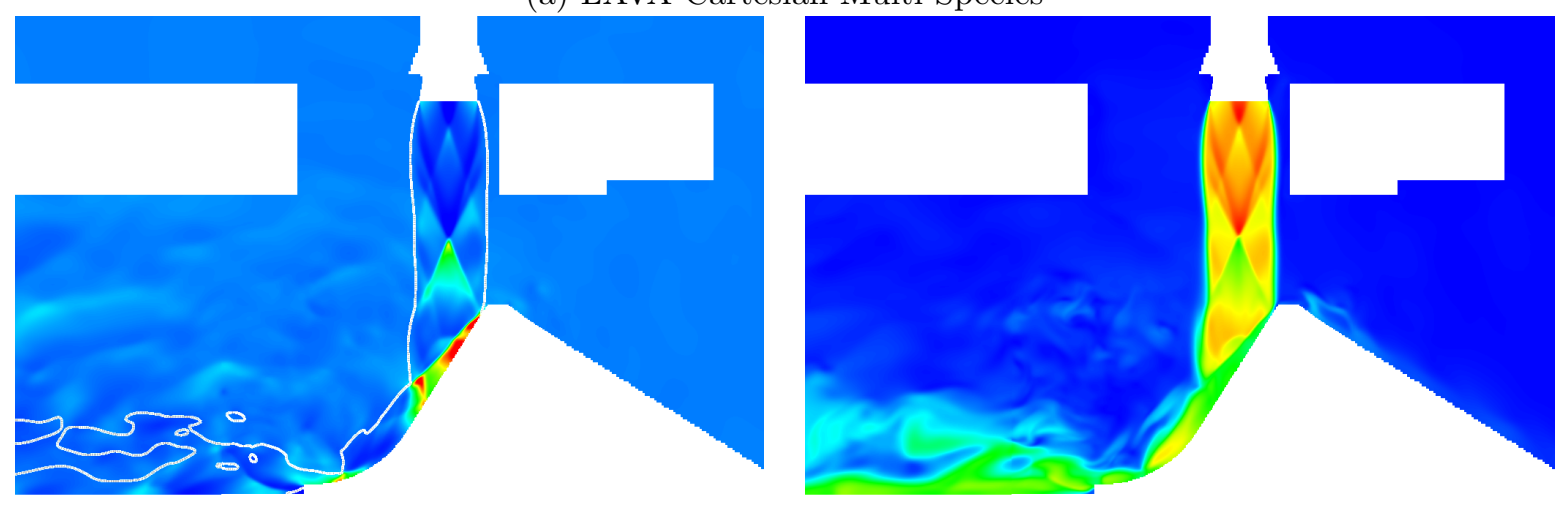

(b) LAVA-Cartesian Single Gas

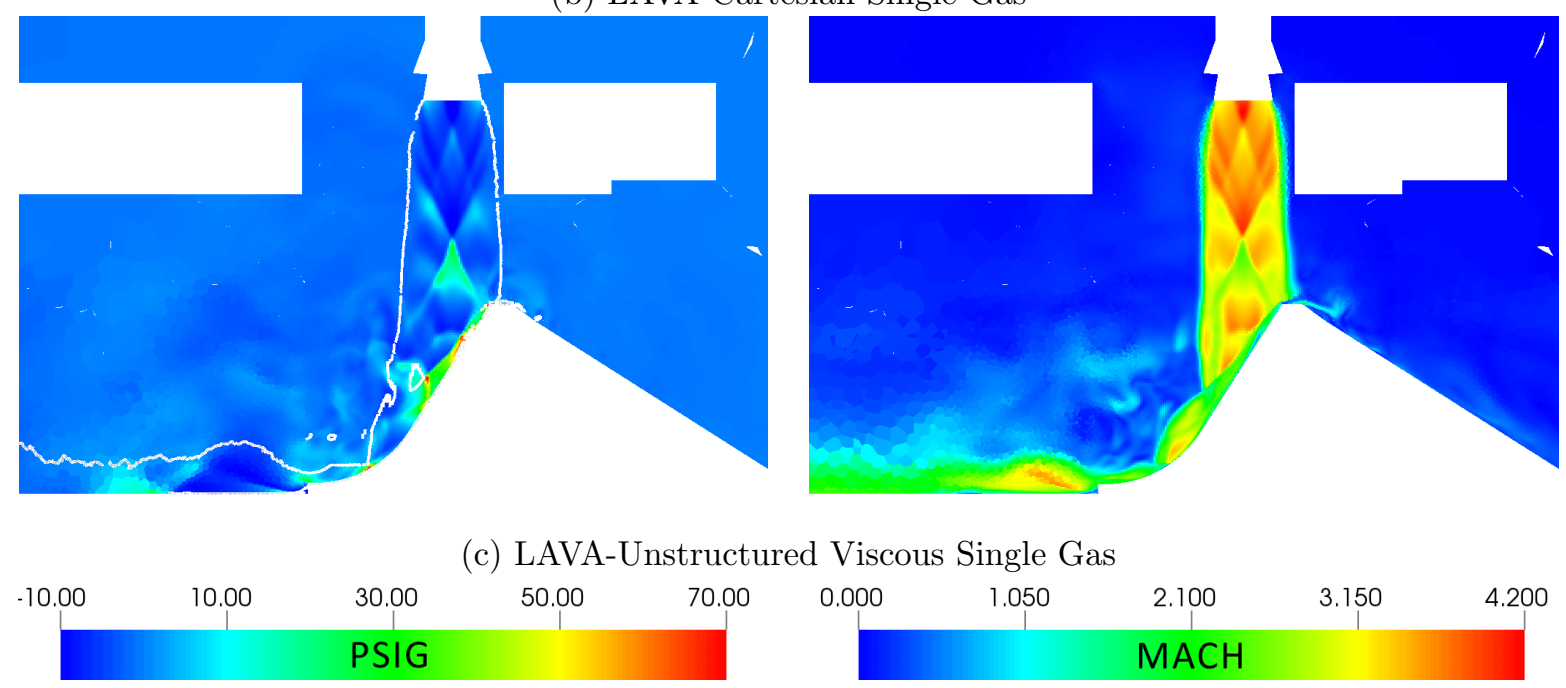

Figure 9. Gauge pressure distributions (PSIG) with white sonic-line contours are shown in the left column with Mach number distributions on the right column at the quasi-steady state full thrust time of $t=0.7$ seconds. From the top to the bottom: (a) inviscid multi-species, (b) inviscid single gas and (c) viscous single gas.

Similar behavior is observed at the middle and bottom sensor location shown in Figure 11 and 12 respectively. Here the different numerical simulations are in good agreement with each other and capture the IOP and pressure signatures. The median value of the flight data appears to be slightly lower, which again can be attributed to the water sound suppression system dampening the pressure field. The unfiltered pressure sensor flight data exhibits a similar IOP pressure amplitude as the simulation data, which is not true of the filtered flight data. 


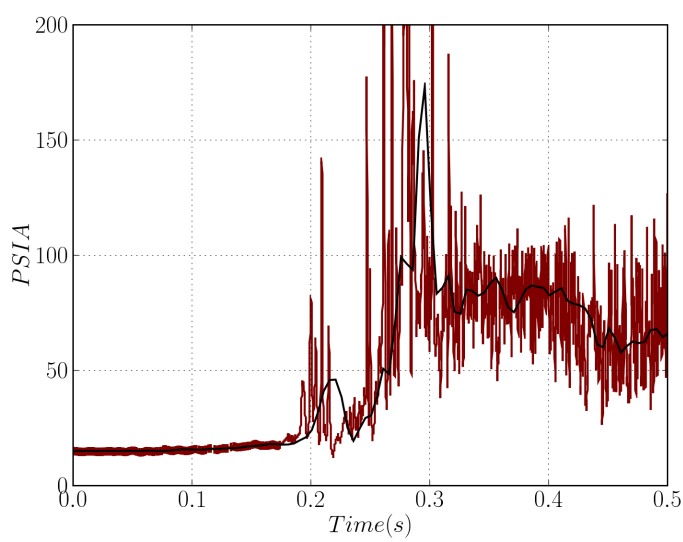

(a) Flight Data

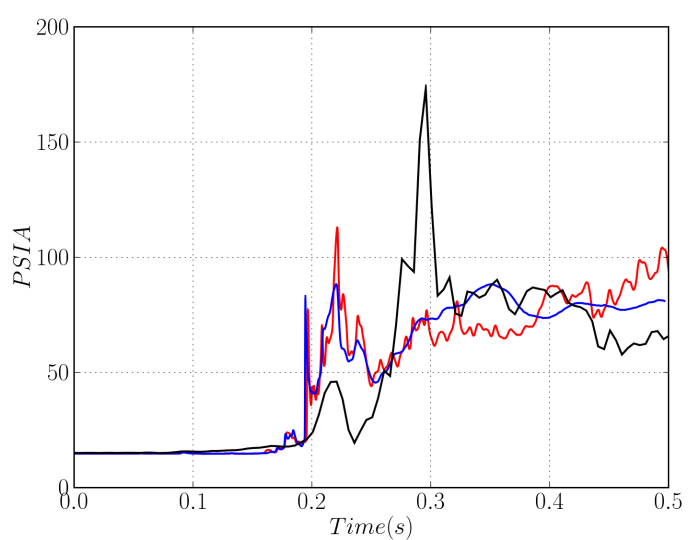

(b) Simulation Data

Figure 10. Unsteady pressure history at top sensor location on MFD. Raw flight data (maroon -), filtered flight data (black -), LAVA-Unstructured viscous (blue -) and LAVA-Cartesian inviscid (red -) are shown.

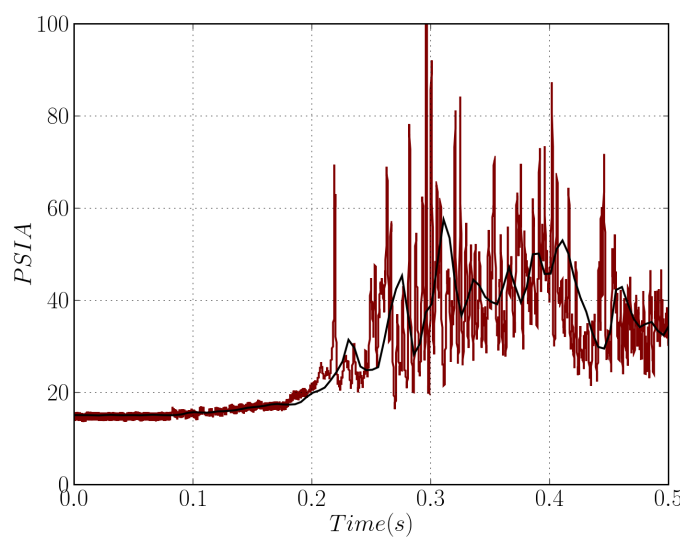

(a) Flight Data

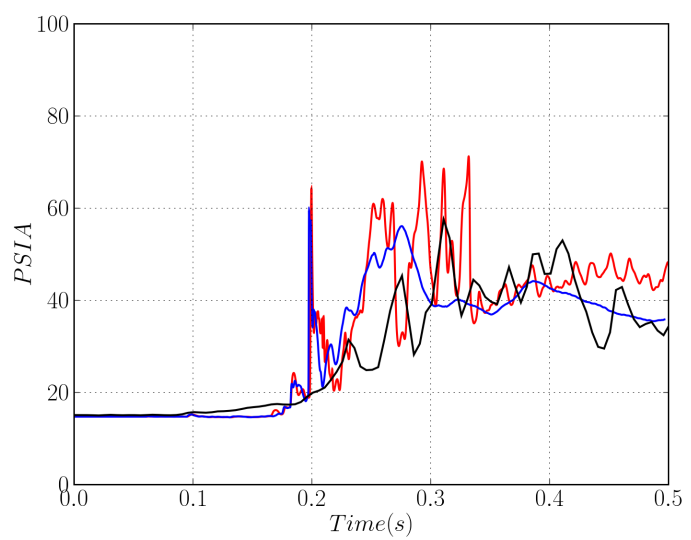

(b) Simulation Data

Figure 11. Unsteady pressure history at middle sensor location on MFD. Raw flight data (maroon -), filtered flight data (black -), LAVA-Unstructured viscous (blue -) and LAVA-Cartesian inviscid (red -) are shown.

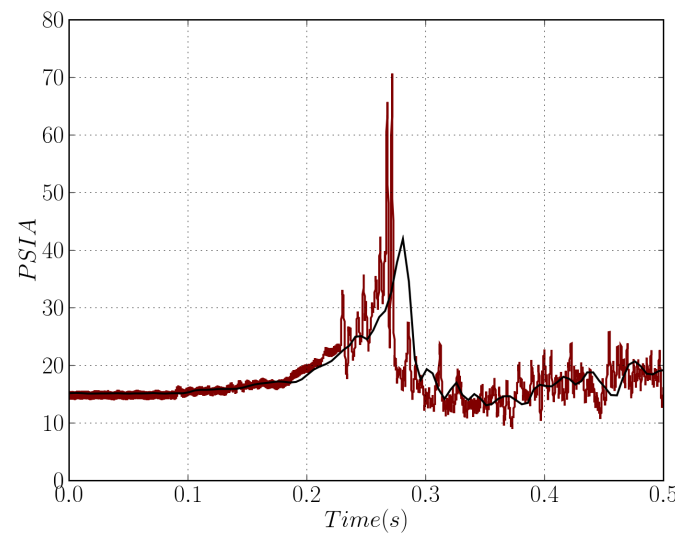

(a) Flight Data

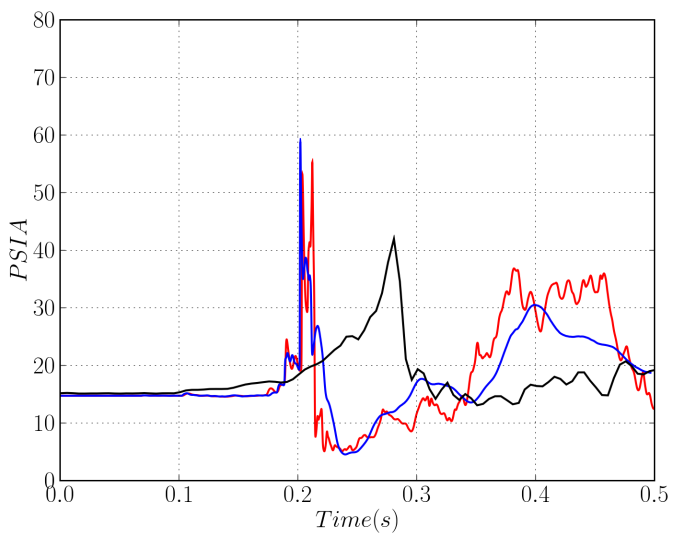

(b) Simulation Data

Figure 12. Unsteady pressure history at bottom sensor location on MFD. Raw flight data (maroon -), filtered flight data (black -), LAVA-Unstructured viscous (blue -) and LAVA-Cartesian inviscid (red -) are shown. 

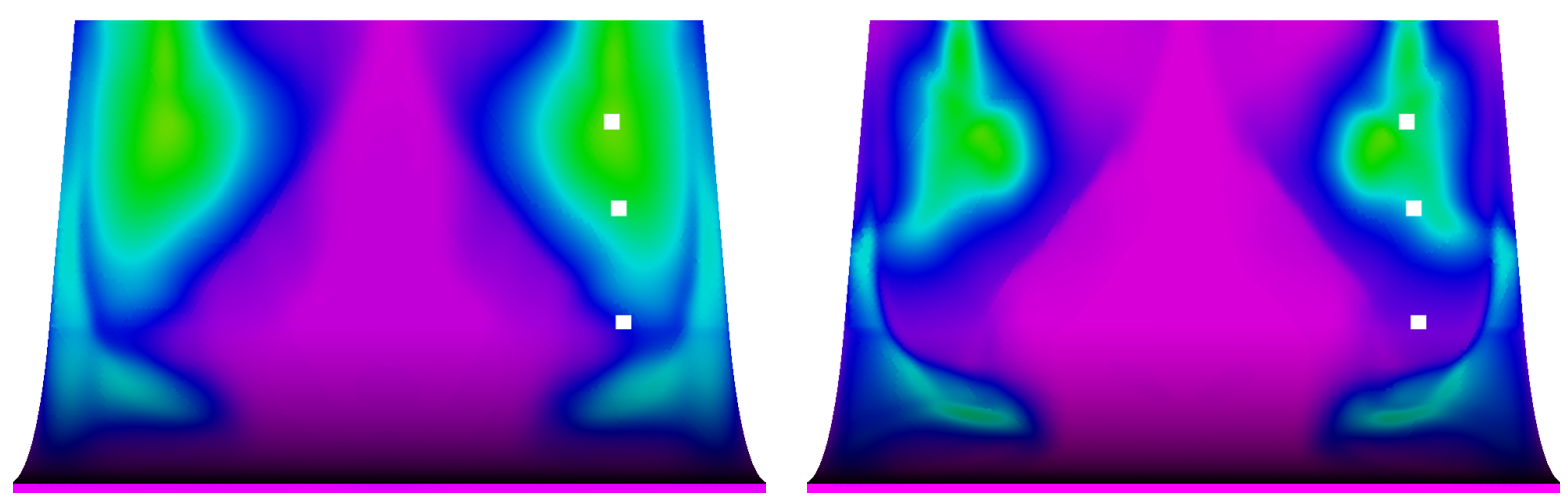

$\mathrm{t}=0.231 \mathrm{~s}$
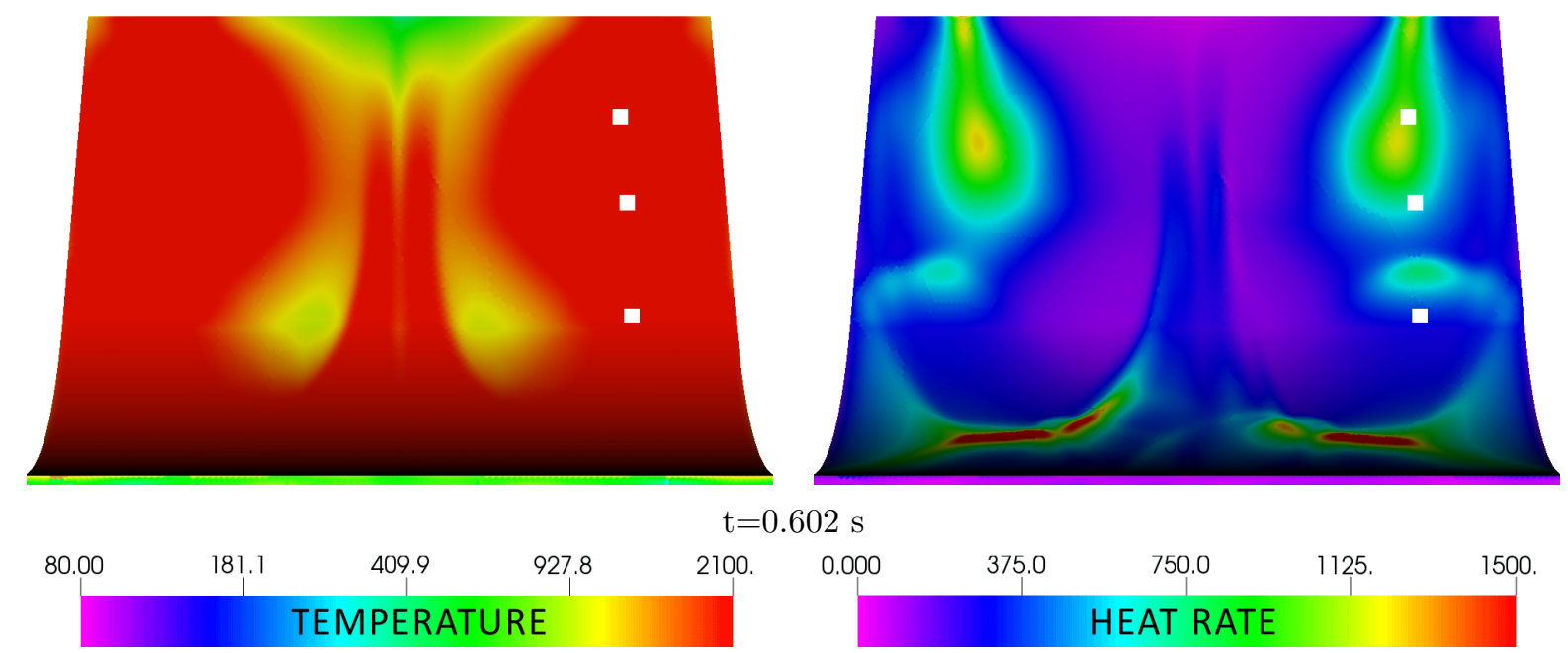

Figure 13. Instantaneous temperature distribution $\left({ }^{\circ} \mathrm{F}\right)$ and heat rate $\left(\mathrm{BTU} / \mathrm{ft}^{2} \mathrm{~s}\right)$ at multiple time sequences using LAVA-Unstructured on the North side of the MFD. The sensor locations are superimposed on the surfaces as white squares.

\section{III.B.4. Results: Thermal Environment}

The thermal environment plays a critical role in the safety and design of the launch site. In particular, thermal protection on the MFD is designed to withstand the high pressure and temperatures of the impinging jet for multiple launches. Insufficient thermal protection can lead to damage of the trench and the potential for debris which may harm the vehicle. Unlike the pressure environment, where inviscid features dominate the flow, viscous simulations are required to accurately capture the viscous heating at the wall. The proposed approach is to use the viscous turbulent capabilities of LAVA-Unstructured with a conjugate heat transfer methodology to approximate wall heating effects. To visualize the thermal environment, the temperature distribution is shown on the MFD in Figure 13.

Comparison of the temperature and heat rate distributions with the pressure distributions shows a strong correlation between high pressure and temperature regions. ${ }^{1}$ Near the first impingement point (top sensor) the surface temperature reaches a local maximum. A strong shock develops on the curved region of the MFD leading to a second impingement point. Along the shock, the temperature field indicates a higher temperature and heat rate region. 


\section{Outlook}

As an example of a launch vehicle SpaceX's Falcon Heavy is used for this analysis. In the following discussion, some preliminary results are shown for Falcon Heavy. The Falcon Heavy configuration consists of a standard Falcon 9 with a payload on top and two additional first stage Falcon 9 used as liquid strap-on boosters.

\section{IV.A. Preliminary Results for Falcon Heavy: Pressure Environment}

Falcon Heavy is a commercial launch vehicle from SpaceX. Simulations were done with 27 Merlin 1C engines. The 27 liquid engines are ignited at $\mathrm{t}=0$. Note that Falcon Heavy may also use Merlin 1-D or Merlin 2 engines. However, only the Merlin 1-C engine data was available to conduct this study. The main flame deflector considered in this analysis is a symmetric flame deflector similar to that used in the Apollo program. For the current analysis of the Falcon Heavy vehicle, 27 Merlin engines at deck-level (Figure 14a) were placed at the SLS North-South location. The unsteady plenum boundary conditions for $T / T_{\max }, p / p_{\text {stag }}$, and $\rho / \rho_{\max }$, where the subscript $\max$ refers to the maximum value and $p_{\text {stag }}$ is the stagnation pressure, are displayed in Figure 14b. The chamber conditions were approximated by scaling a similar LOX/RP1 type engine and matching it with the maximum conditions of Merlin 1C. Quasi-steady state conditions are reached at approximately $t=2 \mathrm{~s}$. The nozzle layout of the 27 Merlin engines are projected onto the MFD in Figures 15.

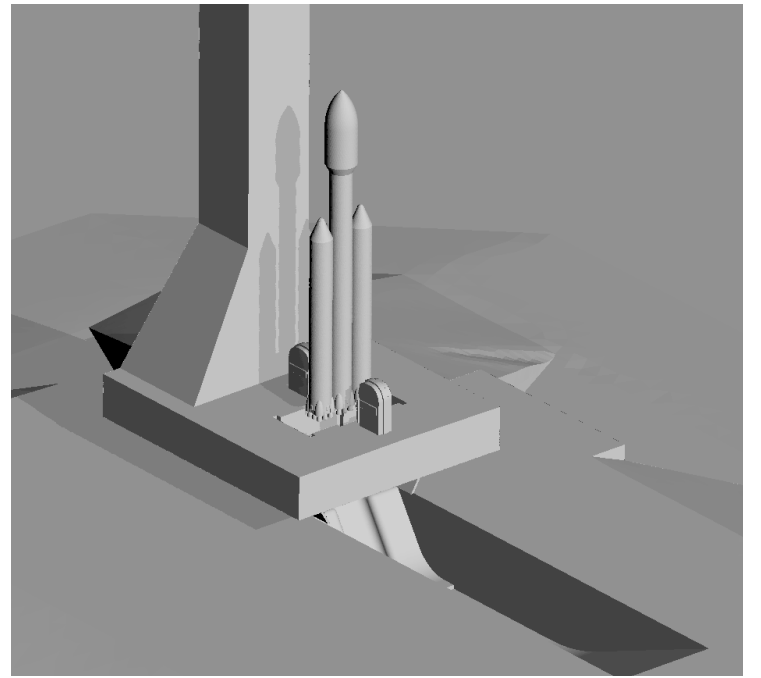

(a)

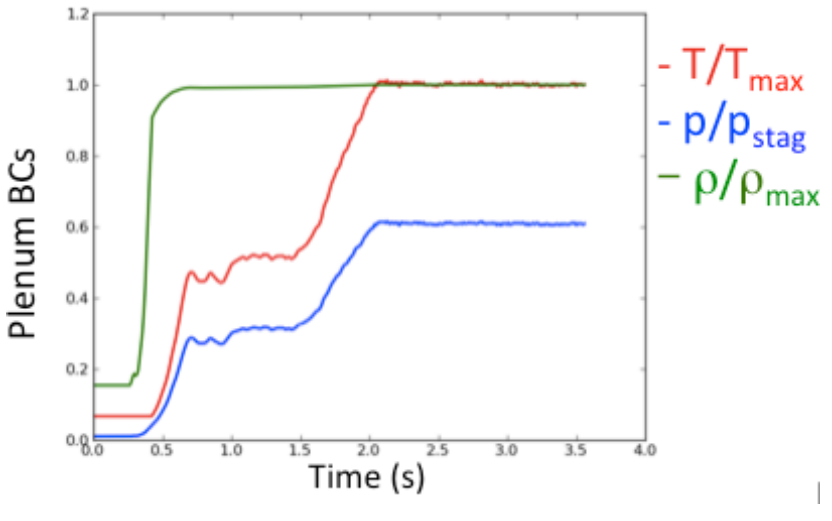

(b)

Figure 14. (a) Falcon Heavy: geometry assembly. (b) Plenum boundary conditions for $T / T_{\max }, p / p_{\text {stag }}$, and $\rho / \rho_{\max }$, where the subscript $\max$ stands for the maximum value and $p_{\text {stag }}$ stands for stagnation pressure.

The computational grid has been refined in regions where large gradients are expected (see view from North in Figure 16a and view from East in Figure 16b) in order to most accurately predict the flow field around the MFDs. Hence, grid points are clustered in the exhaust plume and around the MFD. Adaptive mesh refinement capabilities are available in order to possibly track the propagation of the IOP wave.

The deflector loads are significantly influenced by the impingement angle of the engine plumes. From a simplified inviscid analysis, it is known that when the impingement angle reaches a critical value, the shock wave will detach from the surface which may cause back-flow exhaust towards the vehicle [19]. For the current design analysis, this angle was kept constant based on prior analysis for the Apollo main deflector. The analysis shows that the first impingement of the plume is followed by a secondary impingement. It must be noted that the surface temperatures and pressures at the second impingement location can exceed the values of the primary impingement. Inviscid and viscous CFD simulations are carried out to study the different flow conditions since these highly complex flow structures cannot easily be computed by employing analytical approaches.

The simulation results seem to indicate that the exhaust plume is contained within the trench for the current launch environment configuration. The exhaust plume is visualized by iso-surfaces of total tem- 
perature (Figure 17) with three contour levels, $0.95 T_{t, 0}, 0.50 T_{t, 0}$, and $0.25 T_{t, 0}$, where $T_{t, 0}$ is the full thrust total stagnation temperature at the nozzle exit of the engines. Three snapshots of total temperature are shown during the initial ramp-up of the Merlin engines: 1, 1.5, and 2 seconds after SRB ignition, and a fourth snapshot was taken at 2.5 second after engine ignition when the liquid engines reached quasi-steady state conditions. No significant spill-over of the exhaust plume onto the deck of the flame trench has been recorded for the initial ramp-up phase and during quasi-steady state operation. Due to direct impingement of the Merlin engines onto the apex of the Concept 1C2 MFD, a small fraction of the exhaust plume, at the $0.25 T_{t, 0}$ contour level, deflects to the South side of the Concept 1C2 MFD.

Snapshots of the unsteady pressure distributions on the MFD surface are displayed in Figure 18 for 1, 1.5, 2, and 2.5 seconds after SRB ignition. The pressure signature on the surface of the MFD is highly unsteady. The pressure distributions shortly before $(\mathrm{t}=2.0 \mathrm{~s})$ and during $(\mathrm{t}=2.5 \mathrm{~s})$ quasi-steady state flow conditions are very similar. The unsteady nature of this highly complex flow field will be analyzed in more detail in the final paper.

In order to provide an estimate of the maximum pressure loads we extracted minimum and maximum surface pressure values on the MFD surface over the time interval: $0<t<T_{2}$, where $T_{2}$ is greater than the time $T_{S S}=2 s$ where the quasi-steady state condition is reached. The results of the unsteady pressure analysis are presented in Figure 19 . The pressure ranges from atmospheric pressure $(\approx 14.7$ PSIA $)$ to 130 PSIA for the maximum pressure and from 0.1 PSIA to atmospheric pressure $(\approx 14.7$ PSIA) for the minimum pressure.

The secondary impingement location of the exhaust plume is clearly visible for the Baseline MFD in Fig. 19a. The maximum pressure values in this region are comparable to the pressure values in the primary impingement region. Further, it must be noted that the secondary impingement region is fully covered by the MFD, and thus, no direct impingement on the trench wall can be expected. Local minima in the minimum surface pressure distributions can be seen in between the regions of local maxima in the maximum surface pressure distributions. The spotty minimum surface pressure distribution indicates that the flow field is highly unsteady.

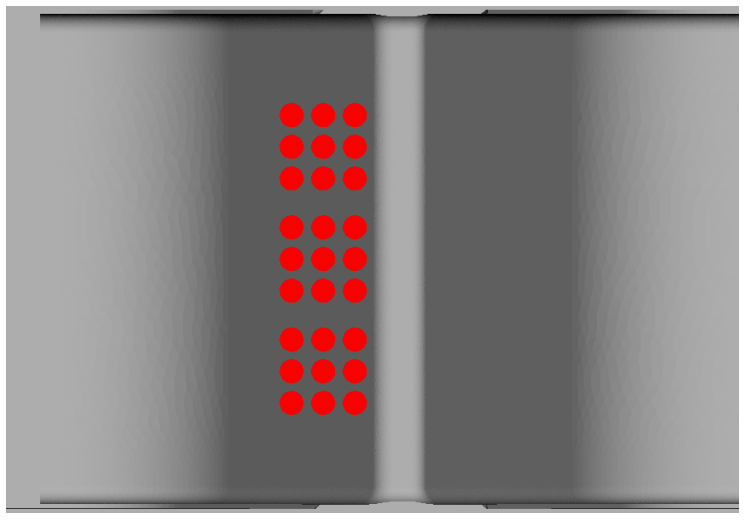

Figure 15. Nozzle layout for Falcon Heavy on MFD. 


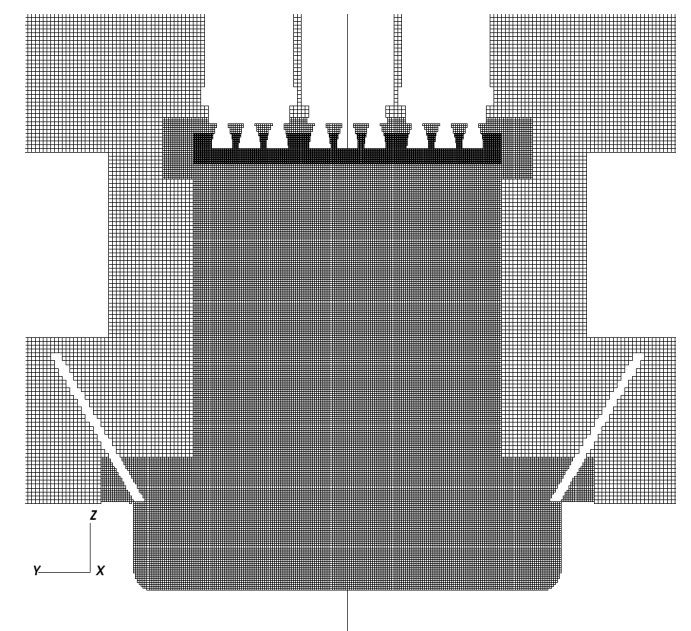

(a)

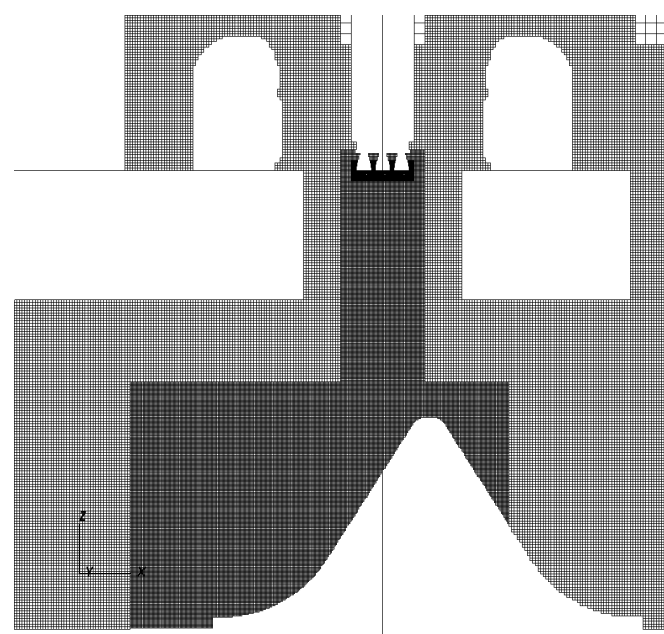

(b)

Figure 16. Volume grid for Falcon Heavy for (a) view from North and (b) view from East.
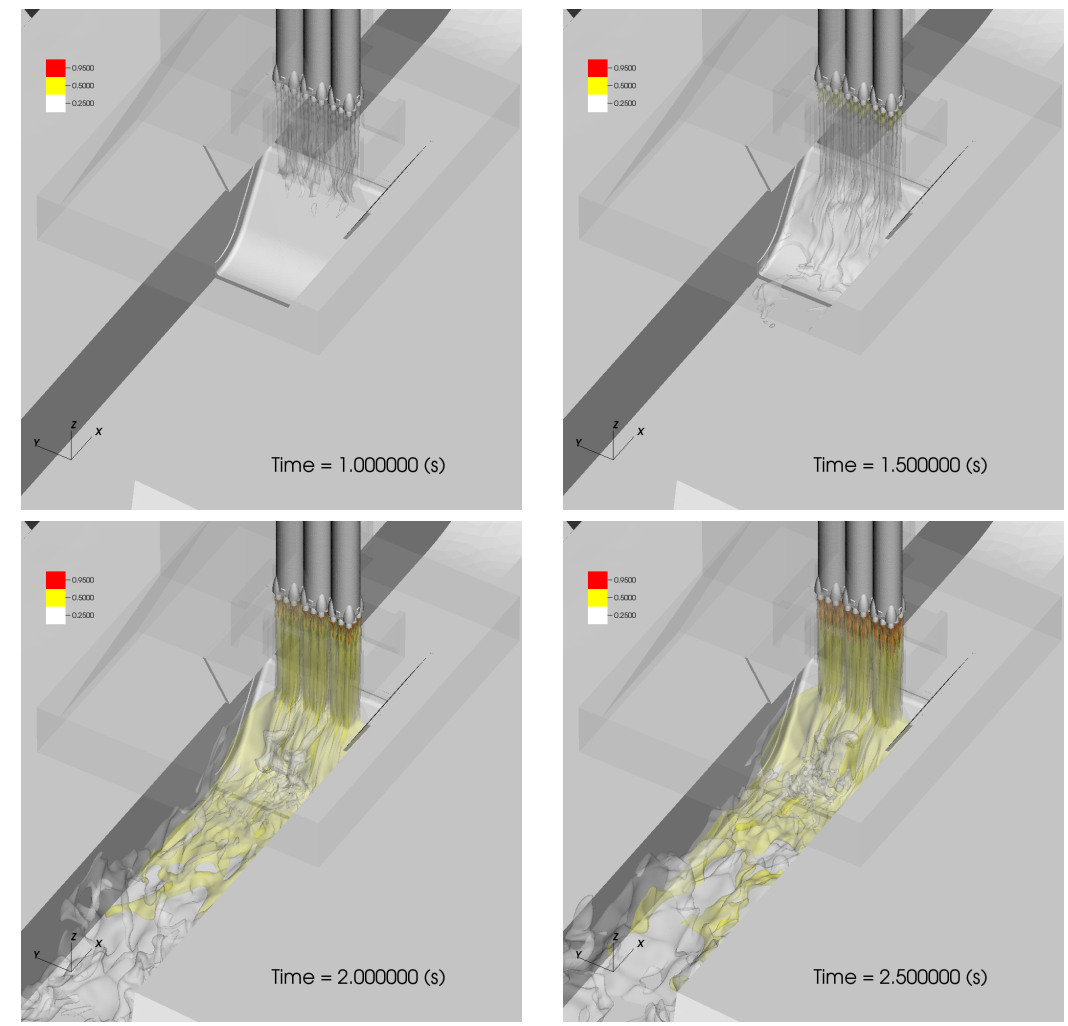

Figure 17. Baseline: Iso-surfaces of total temperature for different MFDs. Red indicates $0.95 T_{t, 0}$, yellow is $0.50 T_{t}, 0$ and white is $0.25 T_{t, 0}$, where $T_{t, 0}$ is the full thrust total stagnation temperature at the nozzle exit of the SRBs. For better visibility the ML is shown as transparent. 

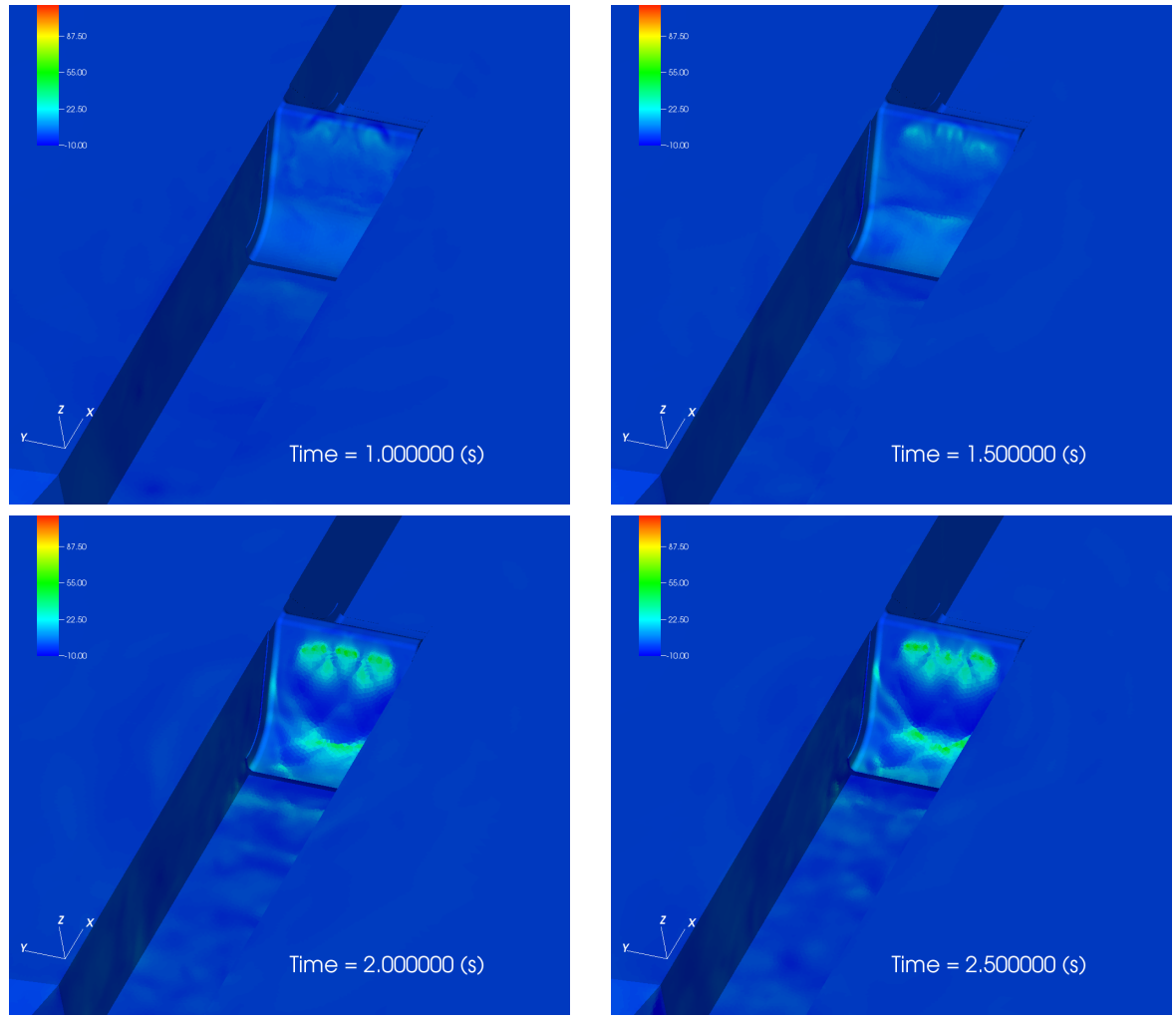

Figure 18. Baseline: Instantaneous pressure distribution (PSIG) at several times using LAVA-Cartesian.

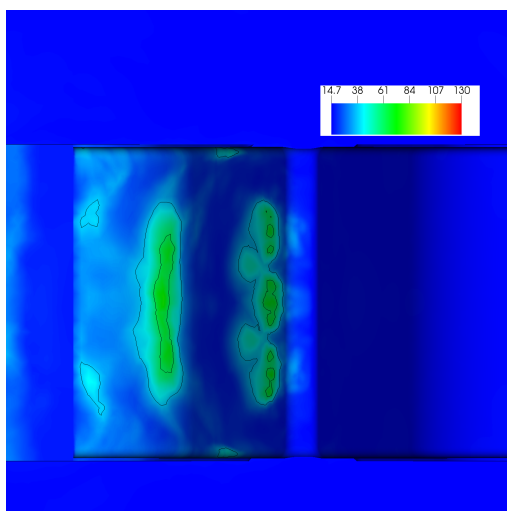

(a) Maximum

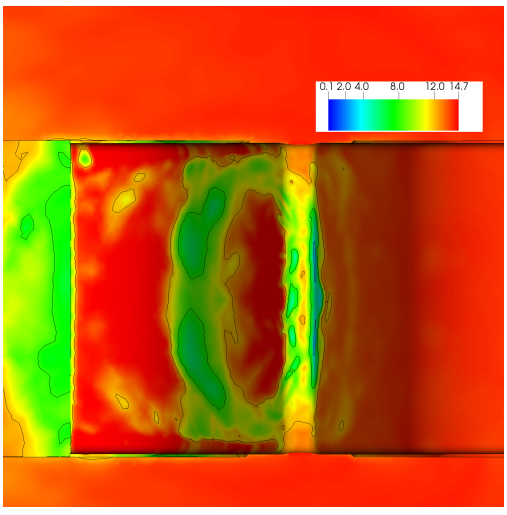

Minimum (b)

Figure 19. Maximum/Minimum surface pressure values over the time interval $0<t<T_{2}$ with $T_{2}>T_{S S}$. $T_{S S}$ is the time where the rocket engines reach quasi-steady state conditions. 

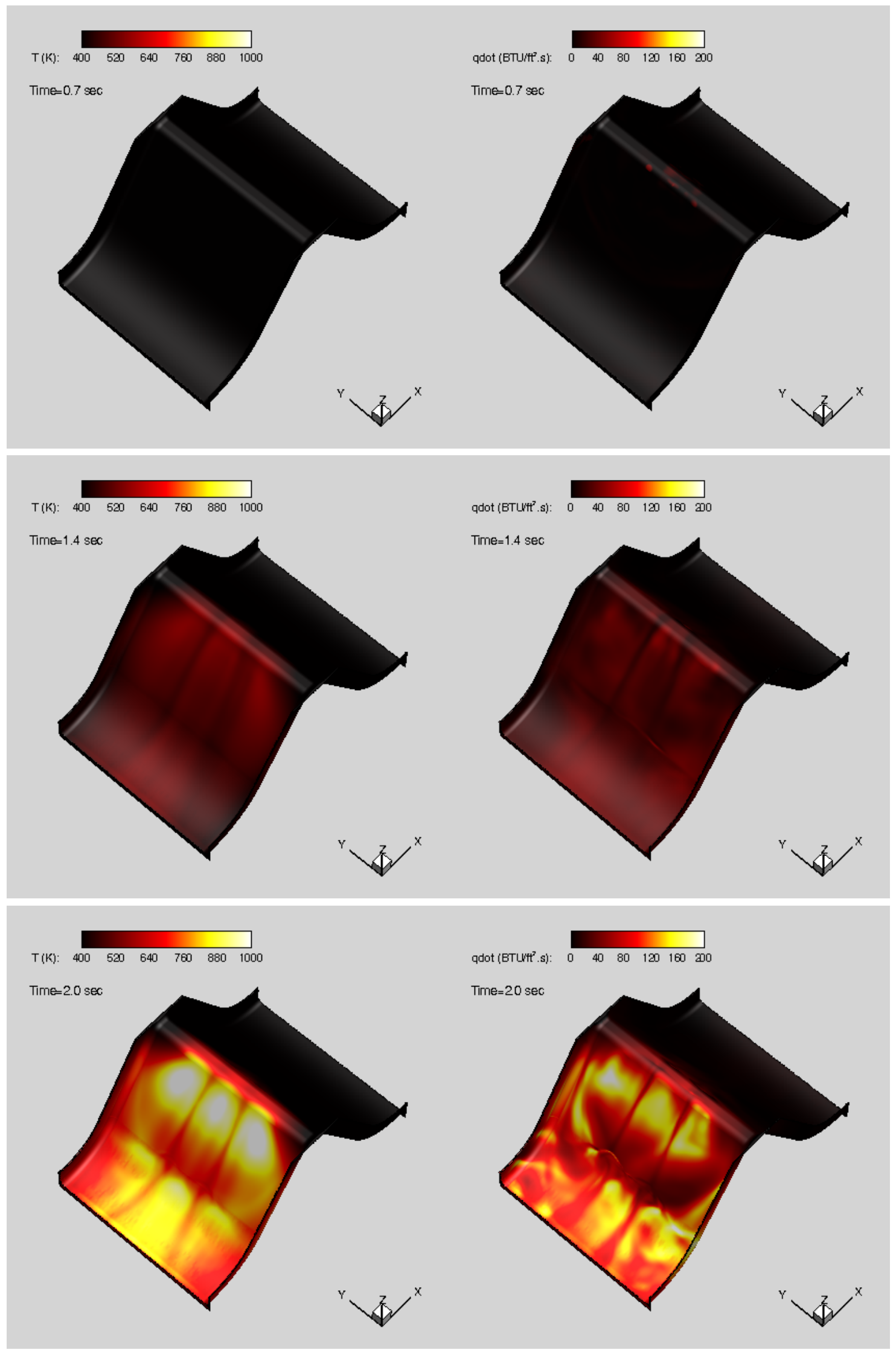

Figure 20. Instantaneous temperature $(L)$ and heat flux $(R)$ distributions for Falcon Heavy vehicle on the Baseline MFD. 


\section{IV.B. Preliminary Results for Falcon Heavy: Thermal Environment}

In the following analysis, the time evolution of temperature and heat flux on the MFD surface and the trench wall are simulated using the LAVA-Unstructured code. The surfaces of interest are assumed to be coated with the Fondu Fyre WA-1 material (see Section §II.B.1 for the material properties used). Unsteady conjugate fluid flow/solid heat conduction simulations are started with the ignition of the engines and followed through the plume development and full thrust phases. Note that the material temperature is not allowed to exceed the given melting point of $1373 \mathrm{~K}$. Validation of the present methodology is presented in. ${ }^{1}$ While the present methodology is shown to yield reasonable predictions, the following needs to be noted:

- Presence of water due to the Ignition Over Pressure (IOP) sound suppression system is neglected. This has been shown ${ }^{19}$ to be a reasonable assumption for the purposes of thermal environment predictions since the water quickly evaporates at the harsh, critical regions of plume impingement.

- Fondu Fyre material is assumed to be dry due to unknown water soaked material properties at the time of simulations.

- Particles from Solid Rocket Motors (SRM), if any, are neglected. The particle effect may be important and needs to be investigated further. However, the validation effort for the STS launch scenario showed conservative predictions even without the particles.

- Radiation is neglected.

- The surface roughness, irregularities, pyrolisis and erosion are neglected.

Viscous grid spacing is included for the MFD, side deflectors and portions of the trench walls and floor. In these regions, the boundary layer is fully resolved with the first grid spacing corresponding to $y+<1$ with a wall-normal grid stretching ratio of 1.2. Simulations are performed with single species gas representing the SRM plume, if any, or the liquid engine plume. Figures 20 shows instantaneous MFD wall temperature and heat flux distributions on the Baseline designs through 2 seconds after ignition. The instantaneous visualization of surface temperature show that the melting temperature is not reached. The main deflector experiences a peak heat flux value of approximately $220 \mathrm{BTU} / \mathrm{ft}^{2} \mathrm{~s}$ near the primary and secondary impingement locations. It must be noted that preliminary analysis of the water sound suppression system showed that water effects are negligible at primary impingement locations. ${ }^{19}$ Similar surface temperatures are reached at the primary impingement location with and without water effects. Away from the direct impingement locations the water effects will lower the surface heat flux.

\section{IV.C. Conclusions}

The current abstract has focused so far on the validation and verification of the employed simulation tools. The final paper will be centered around the actual modeling aspects of the ongoing flow physics in the launch environment. Due to the high degree of unsteadiness in the flow field, Fourier time-series analysis will be necessary to characterize the flow. Additionally, Proper Orthogonal Decomposition (POD) will also be applied in order to extract the most energetic flow structures. The final paper will address the following topics:

- Viscous versus inviscid analysis:

It can be assumed that the effect of the viscous terms in the primary impingement regions are negligible for the pressure signal. At this point it is, however, not quiet clear if the viscous terms greatly affect the location and conditions at the point of secondary impingement. Note that in this section we are mainly focussing on the pressure environmet since the viscous effects are definitely import for the heat transfer.

- Single-species versus multi-species:

It is well-known that the heat-capacity ratio effects the speed-of-sound in the gas. Hence, a change in the frequency spectrum is expected when using a variable heat capacity ratio.

- Calorically perfect gas versus thermally perfect gas:

In the launch environment, a wide range of temperatures are present. Treating the gas as calorically perfect or thermally perfect may not only effect the heat transfer on the main deflector surface but possibly also the topology of the shock structure. 
- Single-engine impingement versus multiple-engine impingement:

It is common practice to approximate the impact of a multiple nozzle configuration as a single nozzle with an "equivalent" nozzle diameter. This model does not account for the interaction between the different jets and leads to misleading results especially regarding condition at the secondary impingement.

- Simplified models to approximate peak pressure and heat flux values:

Since the employed CFD analysis is computationally expensive (hundreds of millions of grid points are used in a single simulation) we will revisit some of the very few analytical tools to estimate the pressure and thermal environments at the impingement locations. These analytical results will be compared to our CFD data and possible improvements to the approximate theory will be proposed.

\section{References}

${ }^{1}$ Moini-Yekta, S., Barad, M., Sozer, E., Brehm, C., Housman, J., and Kiris, C., "Towards Hybrid Grid Simulations of the Launch Environment," ICCFD7-3102, July 9-13, Big Island, Hawaii, 2012.

${ }^{2}$ Berger, M. J. and Colella, P., "Local Adaptive Mesh Refinement for Shock Hydrodynamics," J. Comput. Phys., Vol. 82, No. 1, May 1989, pp. 64-84.

${ }^{3}$ Almgren, A. S., Bell, J. B., Colella, P., Howell, L. H., and Welcome, M. L., "A Conservative Adaptive Projection Method for the Variable Density Incompressible Navier-Stokes Equations," J. Comp. Phys., Vol. 142, 1998, pp. 1-46.

${ }^{4}$ Barad, M. F. and Colella, P., "A Fourth-Order Accurate Local Refinement Method for Poisson's Equation," J. Comp. Phys., Vol. 209, No. 1, October 2005, pp. 1-18.

${ }^{5}$ Barad, M. F., Colella, P., and Schladow, S. G., "An Adaptive Cut-Cell Method for Environmental Fluid Mechanics," Int. J. Numer. Meth. Fluids, Vol. 60, No. 5, 2009, pp. 473-514.

${ }^{6}$ Zhang, Q., Johansen, H., and Colella, P., "A Fourth-Order Accurate Finite-Volume Method with Structured Adaptive Mesh Refinement for Solving the Advection-Diffusion Equation," SIAM Journal on Scientific Computing, Vol. 34, No. 2, 2012, pp. B179-B201.

${ }^{7}$ Colella, P., Graves, D. T., Ligocki, T. J., Martin, D. F., Modiano, D., Serafini, D. B., and Straalen, B. V., "Chombo Software Package for AMR Applications - Design Document," unpublished.

${ }^{8}$ Mittal, R., Dong, H., Bozkurttas, M., Najjar, F., Vargas, A., and von Loebbecke, A., "A versatile sharp interface immersed boundary method for incompressible flows with complex boundaries," Journal of Computational Physics, Vol. 227, No. 10,2008 , pp. $4825-4852$.

${ }^{9}$ Spalart, S. and Allmaras, S., "A One-Equation Turbulence Model for Aerodynamic Flows," 30th Aerospace Sciences Meeting and Exhibit, Reno, NV, January 1992, AIAA-92-0439.

${ }^{10}$ Menter, F., "Zonal Two Equation k- $\omega$ Turbulence Models For Aerodynamic Flows," 23rd Fluid Dynamics, Plasmadynamics, and Lasers Conference, Orlando, FL, July 1993, AIAA-93-2906.

${ }^{11}$ Testing data from Orton Ceramics Materials Testing and Research Center, October 2009.

${ }^{12}$ Buning, P., D.C., J., T.H., P., G.H., K., W.M., C., J.P., S., S.E., K., and K.J., R., "OVERFLOW User's Manual Version 1.8aa," Internal Report NASA Ames Research Center.

${ }^{13}$ Housman, J., Barad, M., Kiris, C., and Kwak, D., "Space-Time Convergence Analysis of a Dual-Time Stepping Method for Simulating Ignition Overpressure Waves," Six International Conference on Computational Fluid Dynamics, to appear, 2010.

${ }^{14}$ Kiris, C., Housman, J., Gusman, M., Chan, W., and Kwak, D., "Time-Accurate Computational Analysis of the Flame Trench Applications," 21st Intl. Conf. on Parallel Computational Fluid Dynamics, 2009, pp. 37-41.

${ }^{15}$ Housman, J., Barad, M., and Kiris, C., "Space-Time Accuracy Assessment of CFD Simulations for the Launch Environment," 29th AIAA Applied Aerodynamics Conference, June 2011, AIAA-2011-3650.

${ }^{16}$ Parlier, C. R., "Pad A Main Flame Deflector Sensor Data and Evaluation," 2011 Thermal and Fluids Analysis Workshop (TFAWS2011), Aug 15-19 2011.

${ }^{17}$ Struchen, L., Trovillion, T., Nerolich, S., Perez, R., Parlier, C., and von Eckroth, W., "Space Shuttle Solid Rocket Motor Plume Pressure and Heat Rate Measurements," 28th Aerodynamic Measurement Technology, Ground Testing, and Flight Testing Conference Conference, June 25-28 2012, AIAA-2012-3293.

${ }^{18}$ Norma, M. L. and Winkler, K.-H. A., "Supersonic Jets," Los Alamos Science, Vol. Sping/Summer, 1985, pp. 38-71.

${ }^{19} \mathrm{Vu}, \mathrm{B} .$, Private communication, 2012. 